Supplement of Hydrol. Earth Syst. Sci. Discuss., 12, 8675-8726, 2015

http://www.hydrol-earth-syst-sci-discuss.net/12/8675/2015/

doi:10.5194/hessd-12-8675-2015-supplement

(C) Author(s) 2015. CC Attribution 3.0 License.

(c) (i)

\title{
A parsimonious analytical model for simulating multispecies plume migration
}

\author{
J.-S. Chen et al. \\ Correspondence to: C.-W. Liu (cwliu@ntu.edu.tw)
}

The copyright of individual parts of the supplement might differ from the CC-BY 3.0 licence. 
Supplement 1. Development of a FORTRAN computer code for the derived analytical solutions in Eqs. (17), (18) and (19)

Note that Eqs. (17)-(19) involve a series of eigenvalues, $\xi_{l}$, which need to be determined from the equation

$\xi_{l} \cot \xi_{l}-\frac{\xi_{l}^{2}}{P e_{L}}+\frac{P e_{L}}{4}=0$

To numerically evaluate these eigenvalues, a bisection method with a small increment of $\xi_{l}$ is used to determine all the eigenvalues. After determining the eigenvalues, we can substitute the eigenvalues into Eqs. (17)-(19) to numerically evaluate the solution. The procedures for numerical evaluation of solution appear to be a straightforward task. Because the solutions in Eqs. (17), (18) and (19) consist of several tiers of summations, we need to use several looping algorithm to achieve the computation of several tiers of summation structure presented in Eqs. (17), (18) and (19). 


\section{Supplement 2. Development of a FORTRAN computer code for the LTFD solution.}

To develop the LTFD solution, a finite difference method is employed by discretizing the $X$ variable of Eqs (A8a) and (A8b). The advection terms are approximated using the upwind difference formulae. After substituting the difference formulae into the transformed partial differential equation, the algebraic equation and rearranging terms, the final form

of the finite difference equations take the following form:

$A_{1}\left(H_{1}\right)_{j-1}+A_{2}\left(H_{1}\right)_{j}+A_{3}\left(H_{1}\right)_{j+1}=A_{4}$

$B_{1}\left(H_{i}\right)_{j-1}+B_{2}\left(H_{i}\right)_{j}+B_{3}\left(H_{i}\right)_{j+1}+B_{4}\left(H_{i-1}\right)_{j}=B_{5} \quad i=2 \ldots, N$

where $A_{1}=\frac{1}{P e_{L}(\Delta X)^{2}}+\frac{1}{\Delta X}, \quad A_{2}=-\frac{2}{P e_{L}(\Delta X)^{2}}-\frac{1}{\Delta X}-\left(R_{1} s+\kappa_{1}+\frac{\rho^{2} n^{2} \pi^{2}}{P e_{T}}\right)$,

$A_{3}=\frac{1}{P e_{L}(\Delta X)^{2}} \quad, \quad A_{4}=0 \quad, \quad B_{1}=\frac{1}{P e_{L}(\Delta X)^{2}}+\frac{1}{\Delta X}$,

$B_{2}=-\frac{2}{P e_{L}(\Delta X)^{2}}-\frac{1}{\Delta X}-\left(R_{i} s+\kappa_{i}+\frac{\rho^{2} n^{2} \pi^{2}}{P e_{T}}\right), \quad B_{3}=\frac{1}{P e_{L}} \frac{1}{(\Delta X)^{2}} \quad, \quad B_{5}=-\kappa_{i-1}$

and $B_{6}=0$.

To solve the coupled set of algebraic equations, several methods have been proposed.

The most rigorous approach is to solve the all algebraic equations simultaneously, which is commonly referred to as the fully-coupled method. Alternatively, iteratively coupled techniques can be employed to obtain the solution. The fully coupled technique requires a global coefficient matrix that includes all the unknown degrees of freedom associated with the discretized problems of the system. However, for most twodimensional coupled differential equation problems, the fully coupled solver is computationally expensive. In contrast, in the sequential iteration approach (SIA), each of the coupled nonlinear differential equation is solved individually in a sequential 
manner, thus greatly reducing the size of the coefficient matrix. Accordingly, the implicit SIA algorithm is adopted in this study to solve the coupled set of algebraic equations. The set of algebraic equations, as represented by Eq. (S2) and (S3) can be solved using direct Gaussian elimination solvers to yield the solution at each node. In addition, a FORTRAN subroutine DLSACB can be used (see Visual Numerics Inc., 1994). The solutions in original domain at each node are the Laplace and finite Fourier cosine inversions of the solution at each node. Finite Fourier cosine inverse transform as in (A12) expressing as an infinite series can be straightforwardly evaluated. However, the Laplace inverse transform must be determined numerically. A FORTRAN subroutine DINLAP/INLAP (Visual Numerics Inc., 1994) based on the de Hoog et al. (1982) algorithm, is employed to perform the Laplace inversion.

\section{References}

Visual Numerics, Inc., 1994. IMSL User’s Manual, Houston, TX. 


\section{Supplement 3. Detailed results of the convergence test examples 1 and 2}

\section{Table S1}

Solution convergence at transect of inlet boundary $(x=0)$ for four-species radionuclide transport problem considering simulated domain of $L=250 \mathrm{~m}$, $W=100 \mathrm{~m}$, subject to Bateman-type sources located at $40 \mathrm{~m} \leq y \leq 60 \mathrm{~m}$ for $t=$ 1,000 year $\quad(M=$ number of terms summed for inverse generalized integral transform; $N=$ number of terms summed for inverse finite Fourier cosine transform).

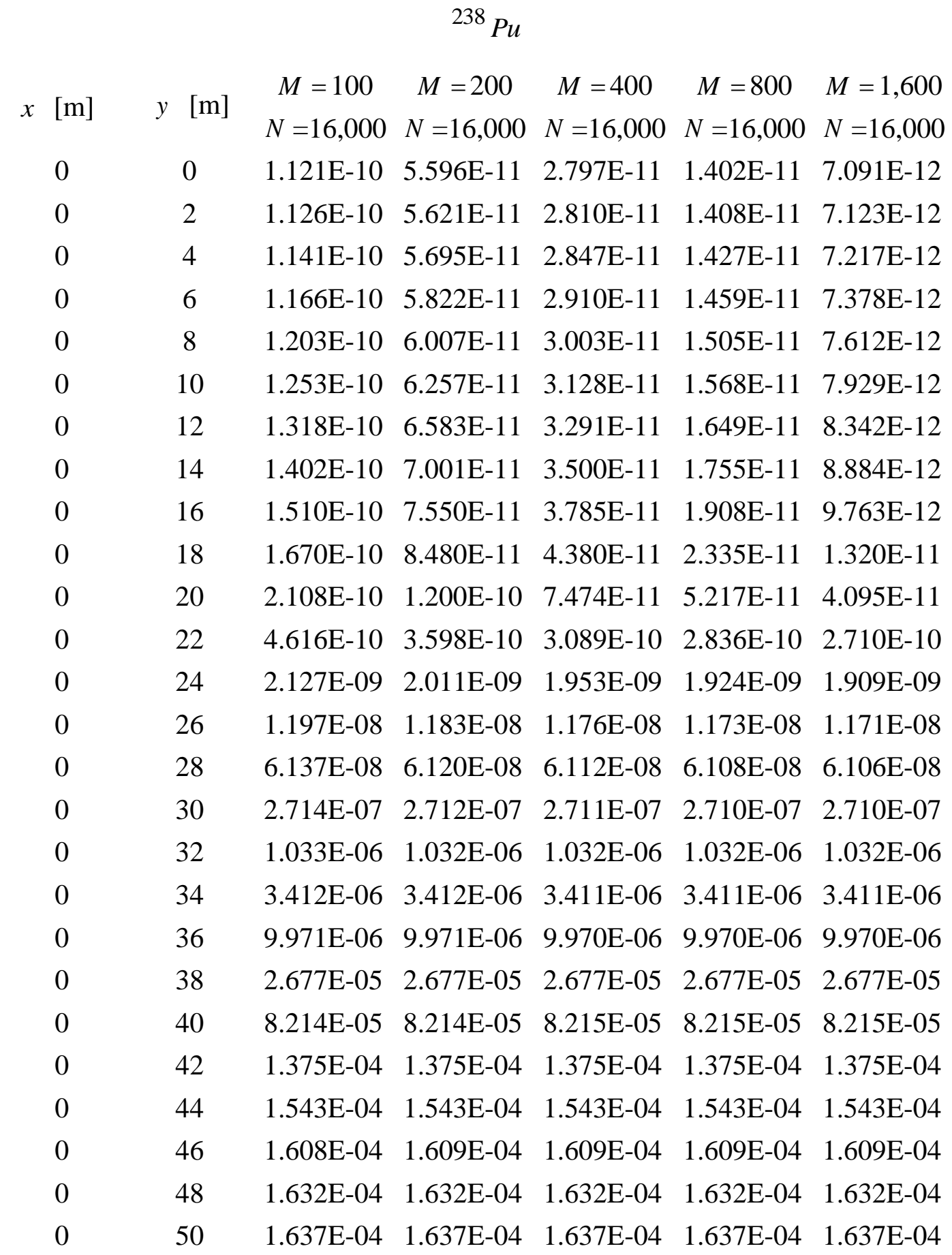


${ }^{238} \mathrm{Pu}$

\begin{tabular}{|c|c|c|c|c|c|c|}
\hline$[\mathrm{m}]$ & $y \quad[\mathrm{~m}]$ & $\begin{aligned} M & =1,600 \\
N & =1,000\end{aligned}$ & $\begin{aligned} M & =1,600 \\
N & =2,000\end{aligned}$ & $\begin{aligned} M & =1,600 \\
N & =4,000\end{aligned}$ & $\begin{aligned} M & =1,600 \\
N & =8,000\end{aligned}$ & $\begin{array}{l}M=1,600 \\
N=16,000\end{array}$ \\
\hline 0 & 0 & $3.612 \mathrm{E}-10$ & $1.003 \mathrm{E}-10$ & $3.451 \mathrm{E}-11$ & $1.483 \mathrm{E}-11$ & 7.09 \\
\hline 0 & 2 & $3.627 \mathrm{E}-10$ & $1.008 \mathrm{E}-10$ & $3.466 \mathrm{E}-11$ & $1.490 \mathrm{E}-11$ & $7.123 \mathrm{E}-12$ \\
\hline 0 & 4 & $3.675 \mathrm{E}-10$ & $1.021 \mathrm{E}-10$ & $3.512 \mathrm{E}-11$ & $1.510 \mathrm{E}-11$ & $7.217 \mathrm{E}-12$ \\
\hline 0 & 6 & $3.757 \mathrm{E}-10$ & $1.044 \mathrm{E}-10$ & $3.590 \mathrm{E}-11$ & $1.543 \mathrm{E}-11$ & 7.37 \\
\hline 0 & 8 & $3.877 \mathrm{E}-10$ & $1.077 \mathrm{E}-10$ & $3.704 \mathrm{E}-11$ & $1.592 \mathrm{E}-11$ & 7.612E-12 \\
\hline 0 & 10 & $4.038 \mathrm{E}-10$ & $1.122 \mathrm{E}-10$ & $3.858 \mathrm{E}-11$ & $1.658 \mathrm{E}-11$ & 7.929E-12 \\
\hline 0 & 12 & $4.248 \mathrm{E}-10$ & $1.180 \mathrm{E}-10$ & $4.059 \mathrm{E}-11$ & $1.745 \mathrm{E}-11$ & 8.342E-12 \\
\hline 0 & 14 & $4.517 \mathrm{E}-10$ & $1.255 \mathrm{E}-10$ & $4.317 \mathrm{E}-11$ & $1.857 \mathrm{E}-11$ & 8.884E-12 \\
\hline 0 & 16 & $4.860 \mathrm{E}-10$ & $1.352 \mathrm{E}-10$ & $4.664 \mathrm{E}-11$ & $2.018 \mathrm{E}-11$ & $9.763 \mathrm{E}-12$ \\
\hline 0 & 18 & $5.319 \mathrm{E}-10$ & $1.498 \mathrm{E}-10$ & $5.337 \mathrm{E}-11$ & $2.454 \mathrm{E}-11$ & $1.320 \mathrm{E}-11$ \\
\hline 0 & 20 & $6.138 \mathrm{E}-10$ & $1.918 \mathrm{E}-10$ & $8.532 \mathrm{E}-11$ & $5.348 \mathrm{E}-11$ & $4.095 \mathrm{E}-11$ \\
\hline 0 & 22 & $9.139 \mathrm{E}-10$ & $4.403 \mathrm{E}-10$ & $3.208 \mathrm{E}-10$ & $2.851 \mathrm{E}-10$ & $2.710 \mathrm{E}-10$ \\
\hline 0 & 24 & 2.644E-09 & 2.103E-09 & $1.966 \mathrm{E}-09$ & $1.925 \mathrm{E}-09$ & 1.909E-09 \\
\hline 0 & 26 & $1.257 \mathrm{E}-08$ & $1.194 \mathrm{E}-08$ & $1.178 \mathrm{E}-08$ & $1.173 \mathrm{E}-08$ & $1.171 \mathrm{E}-08$ \\
\hline 0 & 28 & $6.209 \mathrm{E}-08$ & $6.133 \mathrm{E}-08$ & $6.114 \mathrm{E}-08$ & $6.108 \mathrm{E}-08$ & $6.106 \mathrm{E}-08$ \\
\hline 0 & 30 & $2.723 \mathrm{E}-07$ & $2.714 \mathrm{E}-07$ & $2.711 \mathrm{E}-07$ & $2.710 \mathrm{E}-07$ & $2.710 \mathrm{E}-07$ \\
\hline 0 & 32 & $1.034 \mathrm{E}-06$ & $1.032 \mathrm{E}-06$ & $1.032 \mathrm{E}-06$ & $1.032 \mathrm{E}-06$ & $1.032 \mathrm{E}-06$ \\
\hline 0 & 34 & $3.414 \mathrm{E}-06$ & $3.412 \mathrm{E}-06$ & $3.411 \mathrm{E}-06$ & $3.411 \mathrm{E}-06$ & $3.411 \mathrm{E}-06$ \\
\hline 0 & 36 & 9.974E-06 & 9.971E-06 & $9.970 \mathrm{E}-06$ & $9.970 \mathrm{E}-06$ & $9.970 \mathrm{E}-06$ \\
\hline 0 & 38 & $2.677 \mathrm{E}-05$ & $2.677 \mathrm{E}-05$ & $2.677 \mathrm{E}-05$ & $2.677 \mathrm{E}-05$ & $2.677 \mathrm{E}-05$ \\
\hline 0 & 40 & $8.214 \mathrm{E}-05$ & $8.215 \mathrm{E}-05$ & $8.215 \mathrm{E}-05$ & $8.215 \mathrm{E}-05$ & $8.215 \mathrm{E}-05$ \\
\hline 0 & 42 & $1.375 \mathrm{E}-04$ & $1.375 \mathrm{E}-04$ & $1.375 \mathrm{E}-04$ & $1.375 \mathrm{E}-04$ & $1.375 \mathrm{E}-04$ \\
\hline 0 & 44 & $1.543 \mathrm{E}-04$ & $1.543 \mathrm{E}-04$ & $1.543 \mathrm{E}-04$ & $1.543 \mathrm{E}-04$ & $1.543 \mathrm{E}-04$ \\
\hline 0 & 46 & $1.609 \mathrm{E}-04$ & $1.609 \mathrm{E}-04$ & $1.609 \mathrm{E}-04$ & $1.609 \mathrm{E}-04$ & $1.609 \mathrm{E}-04$ \\
\hline 0 & 48 & $1.632 \mathrm{E}-04$ & $1.632 \mathrm{E}-04$ & $1.632 \mathrm{E}-04$ & $1.632 \mathrm{E}-04$ & $1.632 \mathrm{E}-04$ \\
\hline 0 & 50 & $1.637 \mathrm{E}-04$ & $1.637 \mathrm{E}-04$ & $1.637 \mathrm{E}-04$ & $1.637 \mathrm{E}-04$ & $1.637 \mathrm{E}-04$ \\
\hline
\end{tabular}




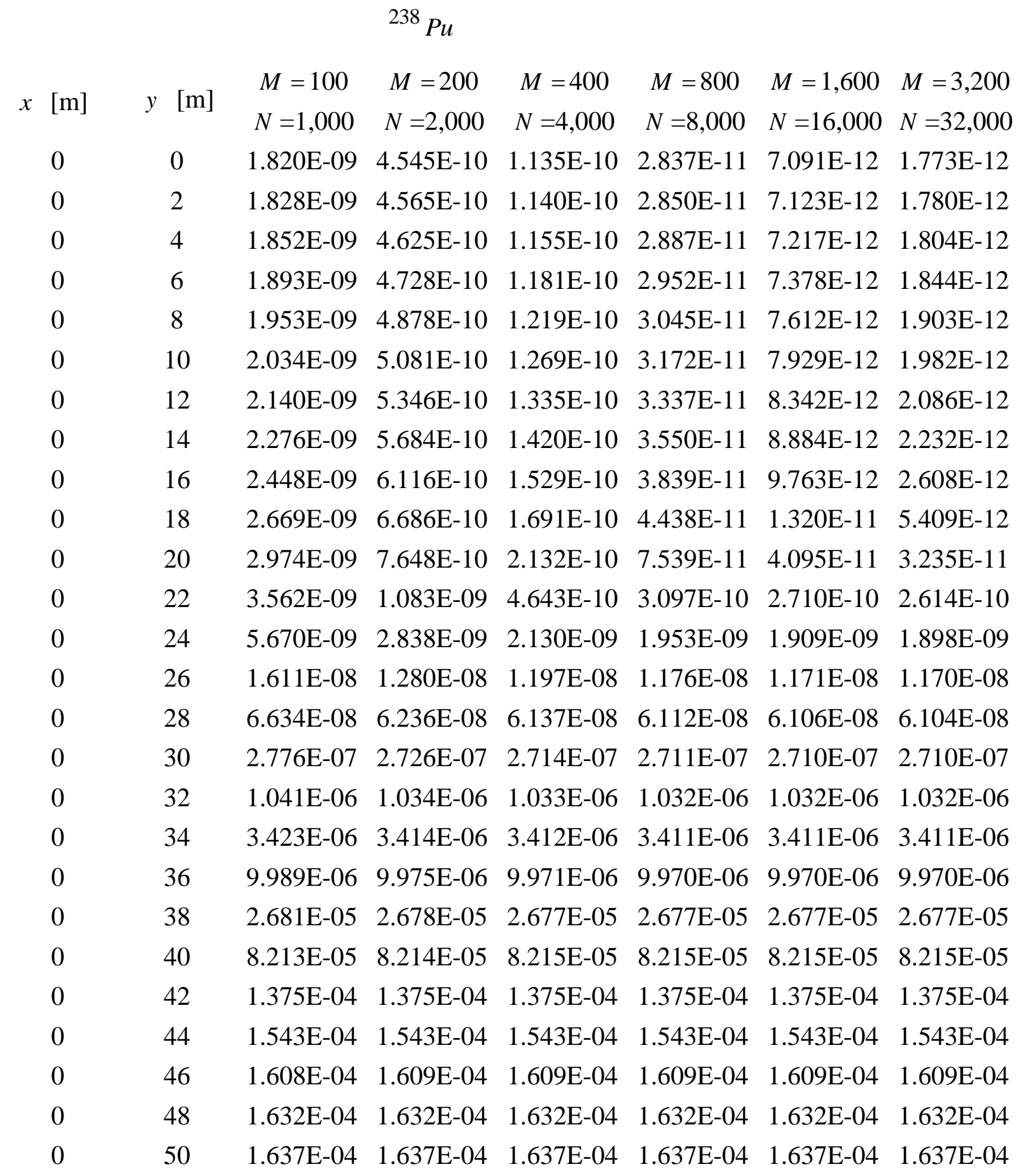




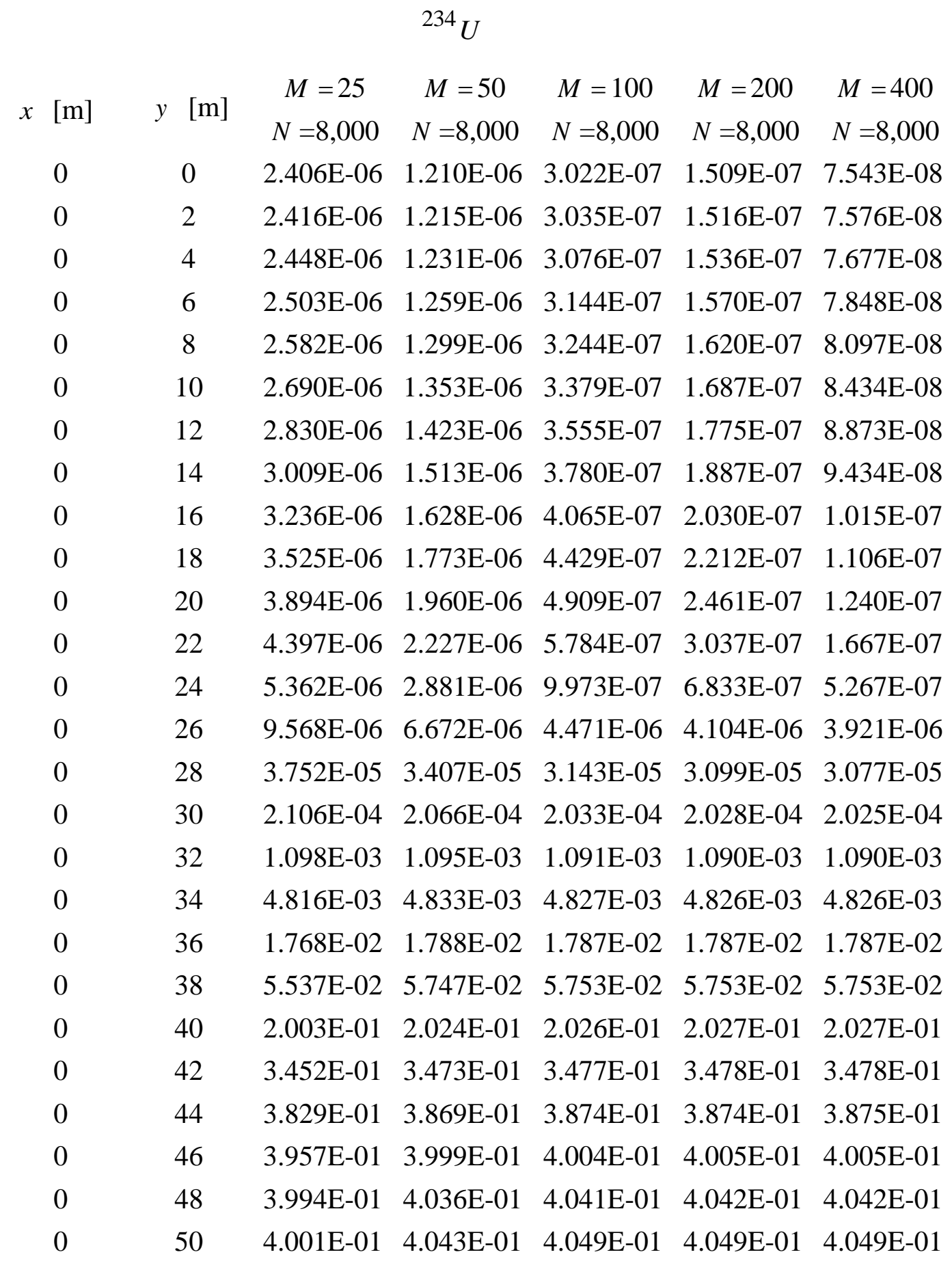




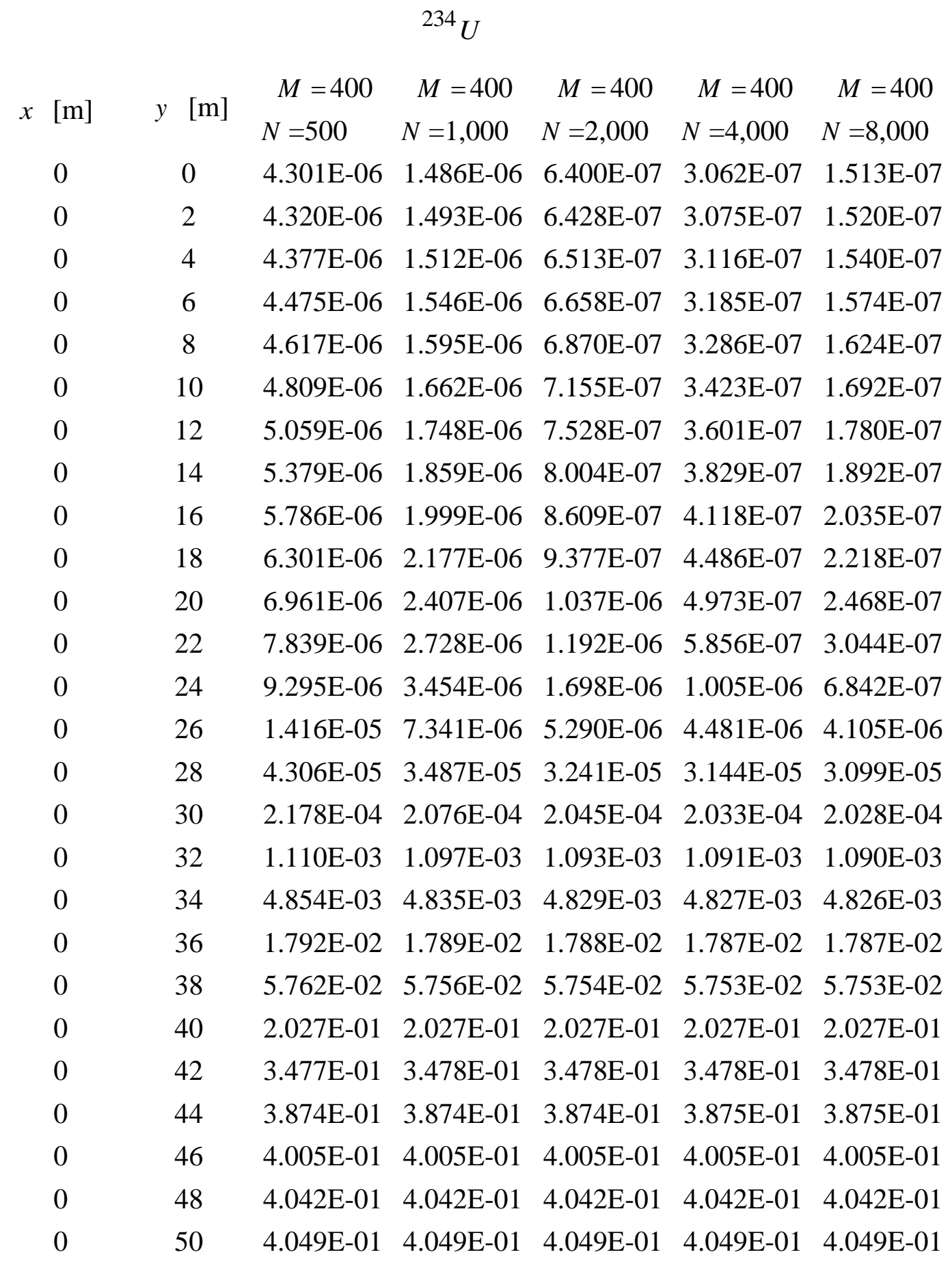




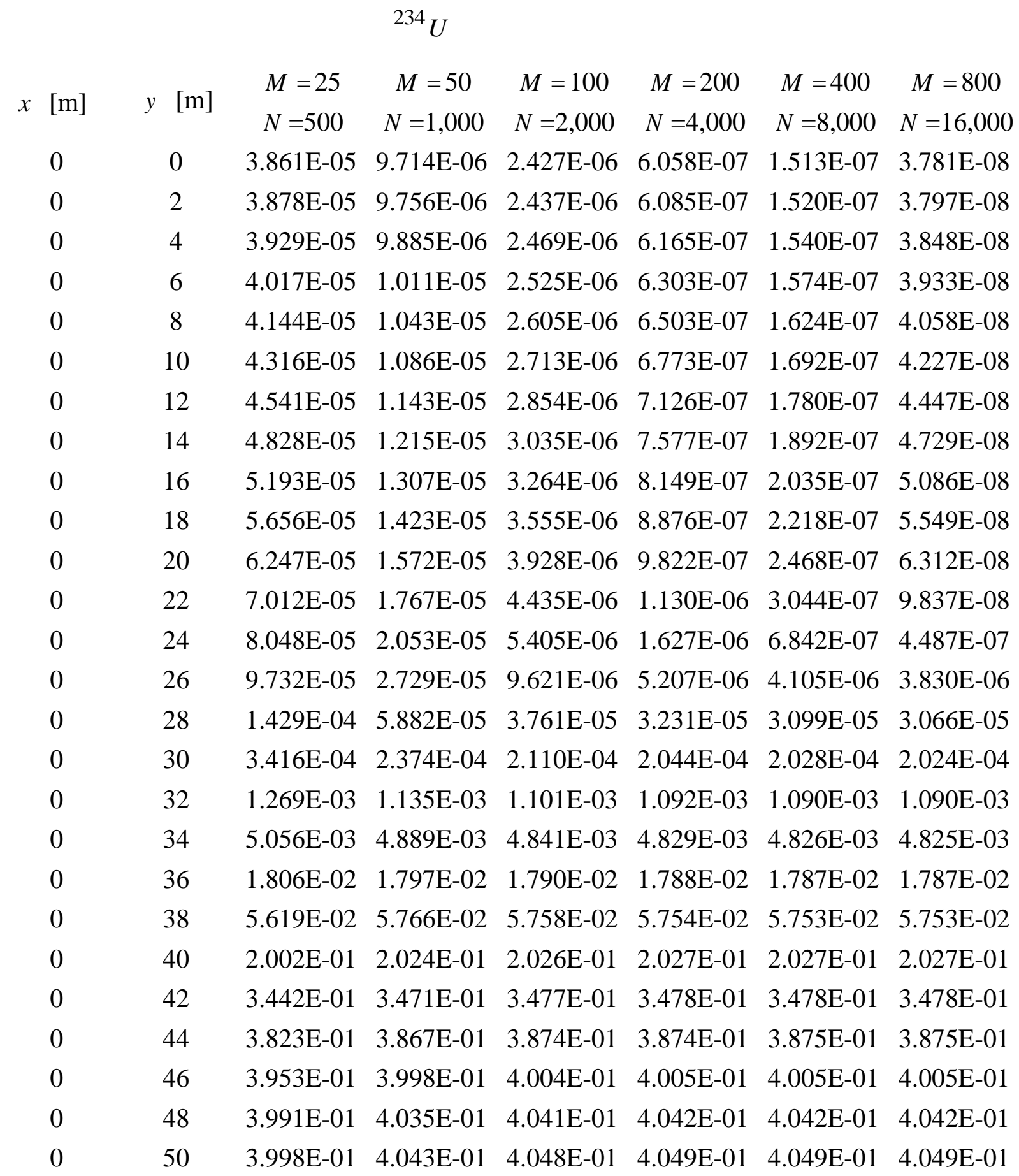




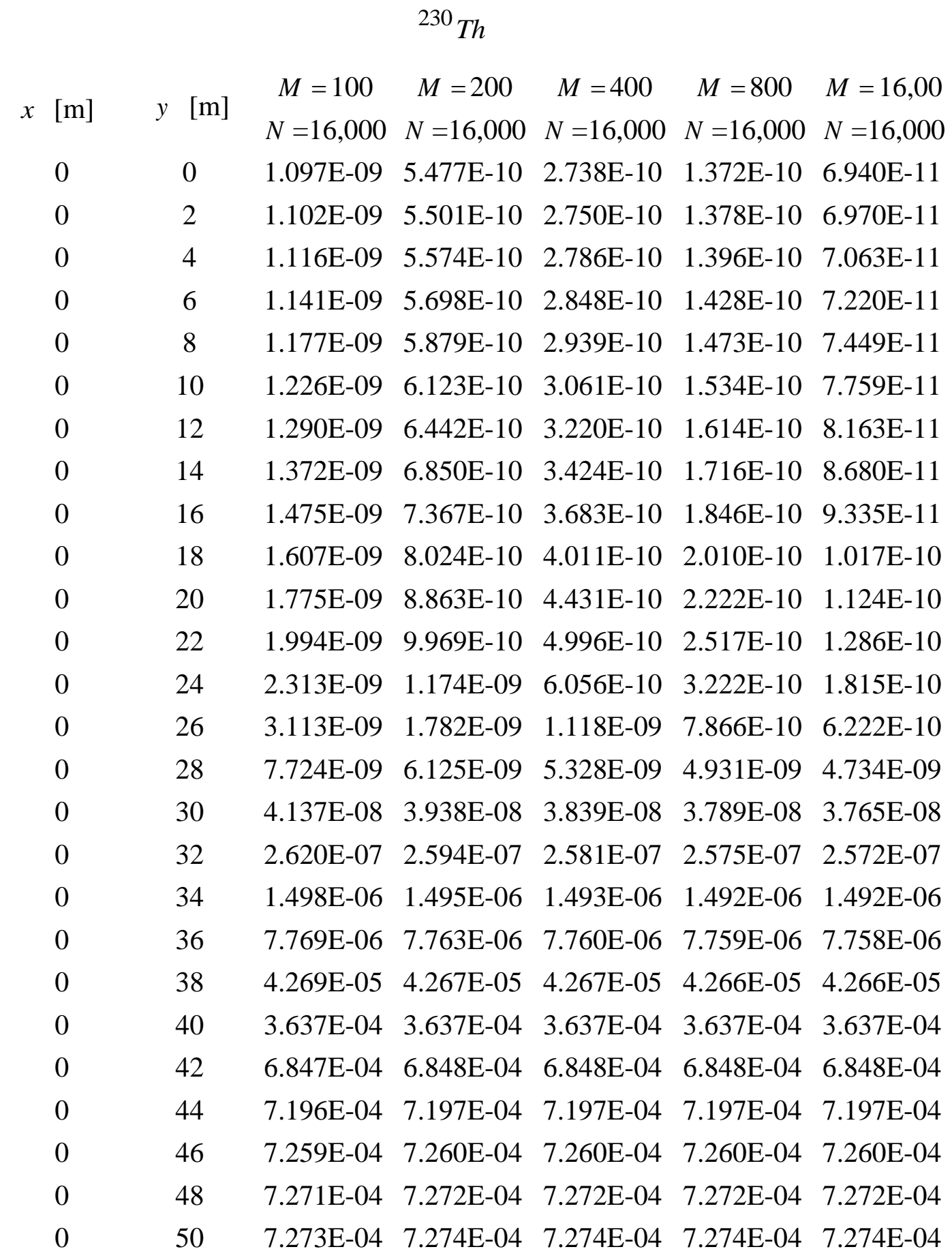


${ }^{230} \mathrm{Th}$

\begin{tabular}{|c|c|c|c|c|c|c|}
\hline \multirow{2}{*}{$x \quad[\mathrm{~m}]$} & \multirow{2}{*}{$y \quad[\mathrm{~m}]$} & $M=1,600$ & $M=1,600$ & $M=1,600$ & $M=1,600$ & $M=1,600$ \\
\hline & & $N=1,000$ & $N=2,000$ & $N=4,000$ & $N=8,000$ & $N=16,000$ \\
\hline 0 & 0 & 3.534E-09 & $9.818 \mathrm{E}-10$ & $3.377 \mathrm{E}-10$ & $1.452 \mathrm{E}-10$ & $6.940 \mathrm{E}-11$ \\
\hline 0 & 2 & $3.550 \mathrm{E}-09$ & $9.861 \mathrm{E}-10$ & $3.392 \mathrm{E}-10$ & $1.458 \mathrm{E}-10$ & $6.970 \mathrm{E}-11$ \\
\hline 0 & 4 & $3.597 \mathrm{E}-09$ & $9.991 \mathrm{E}-10$ & $3.437 \mathrm{E}-10$ & $1.477 \mathrm{E}-10$ & $7.063 \mathrm{E}-11$ \\
\hline 0 & 6 & $3.677 \mathrm{E}-09$ & $1.021 \mathrm{E}-09$ & $3.514 \mathrm{E}-10$ & $1.510 \mathrm{E}-10$ & $7.220 \mathrm{E}-11$ \\
\hline 0 & 8 & 3.794E-09 & $1.054 \mathrm{E}-09$ & $3.625 \mathrm{E}-10$ & $1.558 \mathrm{E}-10$ & 7.449E-11 \\
\hline 0 & 10 & 3.952E-09 & 1.098E-09 & $3.776 \mathrm{E}-10$ & $1.623 \mathrm{E}-10$ & $7.759 \mathrm{E}-11$ \\
\hline 0 & 12 & 4.157E-09 & $1.155 \mathrm{E}-09$ & $3.972 \mathrm{E}-10$ & $1.707 \mathrm{E}-10$ & $8.163 \mathrm{E}-11$ \\
\hline 0 & 14 & 4.420E-09 & $1.228 \mathrm{E}-09$ & $4.224 \mathrm{E}-10$ & $1.815 \mathrm{E}-10$ & $8.680 \mathrm{E}-11$ \\
\hline 0 & 16 & 4.754E-09 & $1.321 \mathrm{E}-09$ & $4.543 \mathrm{E}-10$ & $1.953 \mathrm{E}-10$ & $9.335 \mathrm{E}-11$ \\
\hline 0 & 18 & 5.178E-09 & $1.438 \mathrm{E}-09$ & $4.948 \mathrm{E}-10$ & $2.127 \mathrm{E}-10$ & $1.017 \mathrm{E}-10$ \\
\hline 0 & 20 & 5.719E-09 & $1.589 \mathrm{E}-09$ & $5.466 \mathrm{E}-10$ & $2.350 \mathrm{E}-10$ & $1.124 \mathrm{E}-10$ \\
\hline 0 & 22 & 6.419E-09 & $1.785 \mathrm{E}-09$ & $6.157 \mathrm{E}-10$ & $2.661 \mathrm{E}-10$ & $1.286 \mathrm{E}-10$ \\
\hline 0 & 24 & 7.371E-09 & $2.075 \mathrm{E}-09$ & 7.383E-10 & $3.387 \mathrm{E}-10$ & $1.815 \mathrm{E}-10$ \\
\hline 0 & 26 & $9.022 \mathrm{E}-09$ & 2.834E-09 & $1.273 \mathrm{E}-09$ & $8.059 \mathrm{E}-10$ & $6.222 \mathrm{E}-10$ \\
\hline 0 & 28 & $1.482 \mathrm{E}-08$ & 7.389E-09 & $5.514 \mathrm{E}-09$ & 4.954E-09 & 4.734E-09 \\
\hline 0 & 30 & $5.018 \mathrm{E}-08$ & $4.095 \mathrm{E}-08$ & $3.862 \mathrm{E}-08$ & 3.792E-08 & $3.765 \mathrm{E}-08$ \\
\hline 0 & 32 & $2.735 \mathrm{E}-07$ & $2.615 \mathrm{E}-07$ & $2.584 \mathrm{E}-07$ & $2.575 \mathrm{E}-07$ & $2.572 \mathrm{E}-07$ \\
\hline 0 & 34 & $1.515 \mathrm{E}-06$ & $1.498 \mathrm{E}-06$ & $1.493 \mathrm{E}-06$ & $1.492 \mathrm{E}-06$ & $1.492 \mathrm{E}-06$ \\
\hline 0 & 36 & 7.794E-06 & 7.768E-06 & 7.761E-06 & 7.759E-06 & $7.758 \mathrm{E}-06$ \\
\hline 0 & 38 & 4.274E-05 & $4.268 \mathrm{E}-05$ & $4.267 \mathrm{E}-05$ & 4.267E-05 & $4.266 \mathrm{E}-05$ \\
\hline 0 & 40 & $3.637 \mathrm{E}-04$ & $3.637 \mathrm{E}-04$ & $3.637 \mathrm{E}-04$ & $3.637 \mathrm{E}-04$ & $3.637 \mathrm{E}-04$ \\
\hline 0 & 42 & $6.847 \mathrm{E}-04$ & $6.848 \mathrm{E}-04$ & $6.848 \mathrm{E}-04$ & $6.848 \mathrm{E}-04$ & $6.848 \mathrm{E}-04$ \\
\hline 0 & 44 & 7.197E-04 & 7.197E-04 & 7.197E-04 & 7.197E-04 & 7.197E-04 \\
\hline 0 & 46 & 7.259E-04 & 7.260E-04 & $7.260 \mathrm{E}-04$ & $7.260 \mathrm{E}-04$ & $7.260 \mathrm{E}-04$ \\
\hline 0 & 48 & 7.272E-04 & 7.272E-04 & $7.272 \mathrm{E}-04$ & 7.272E-04 & 7.272E-04 \\
\hline 0 & 50 & 7.274E-04 & 7.274E-04 & $7.274 \mathrm{E}-04$ & 7.274E-04 & 7.274E-04 \\
\hline
\end{tabular}




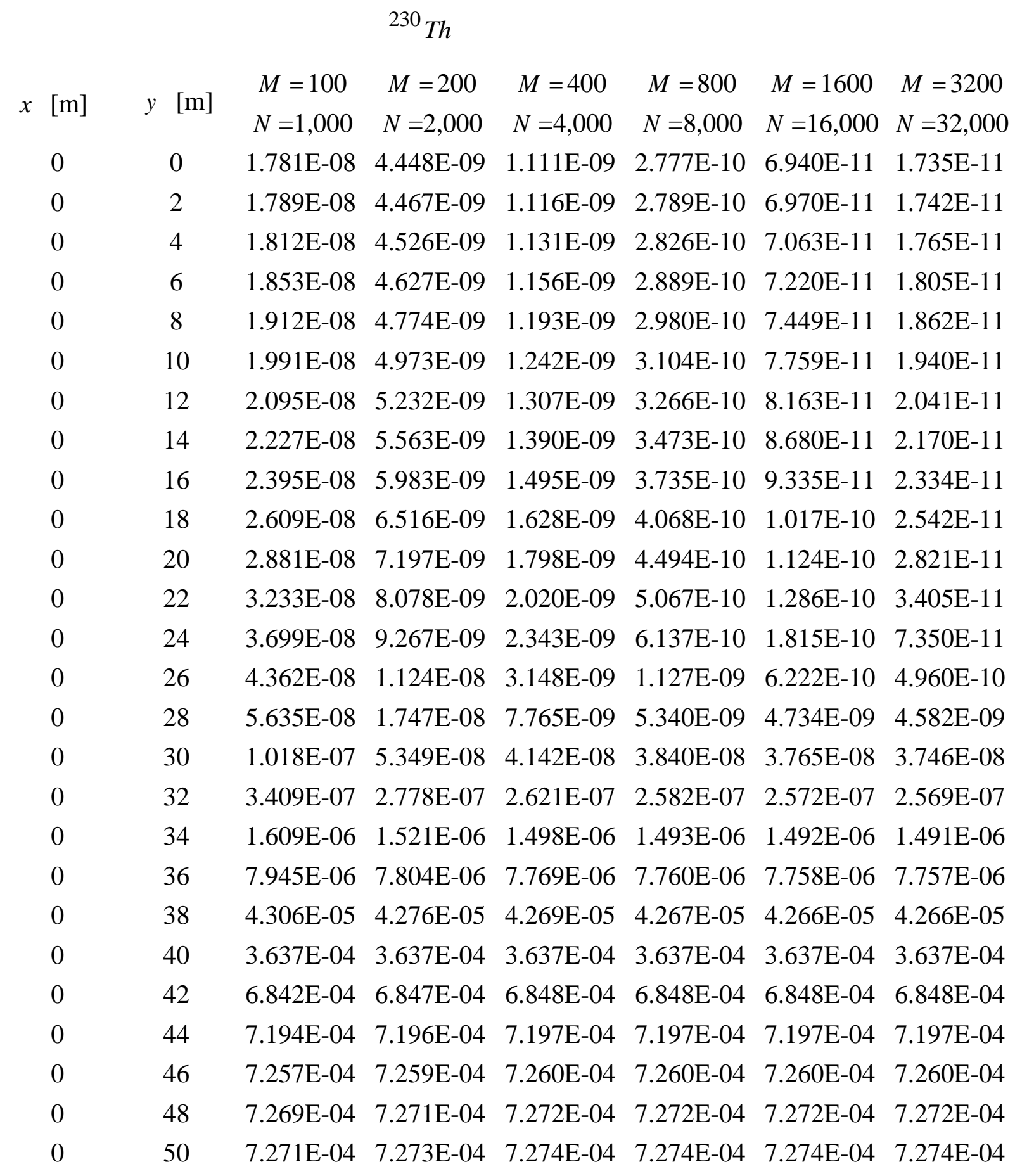




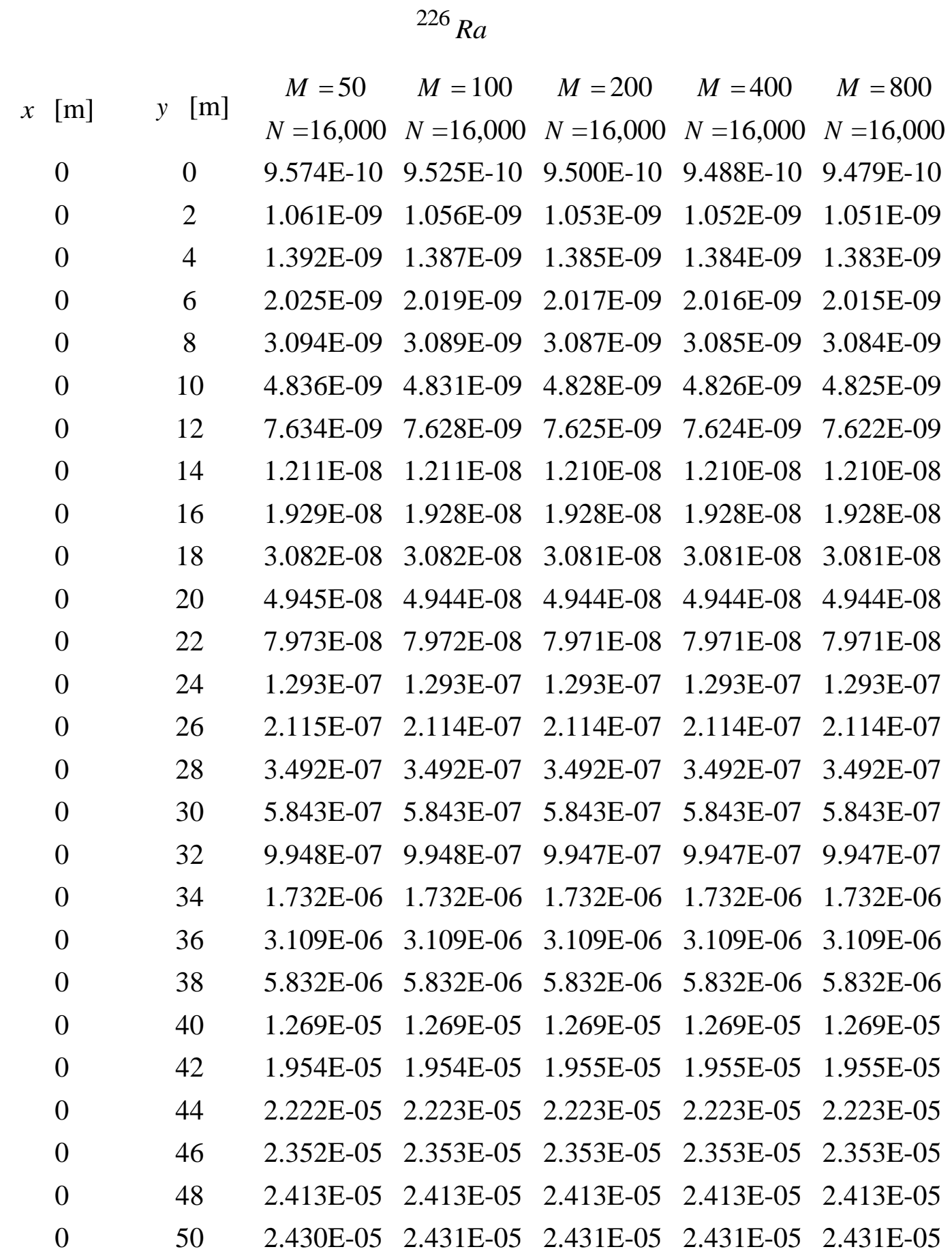




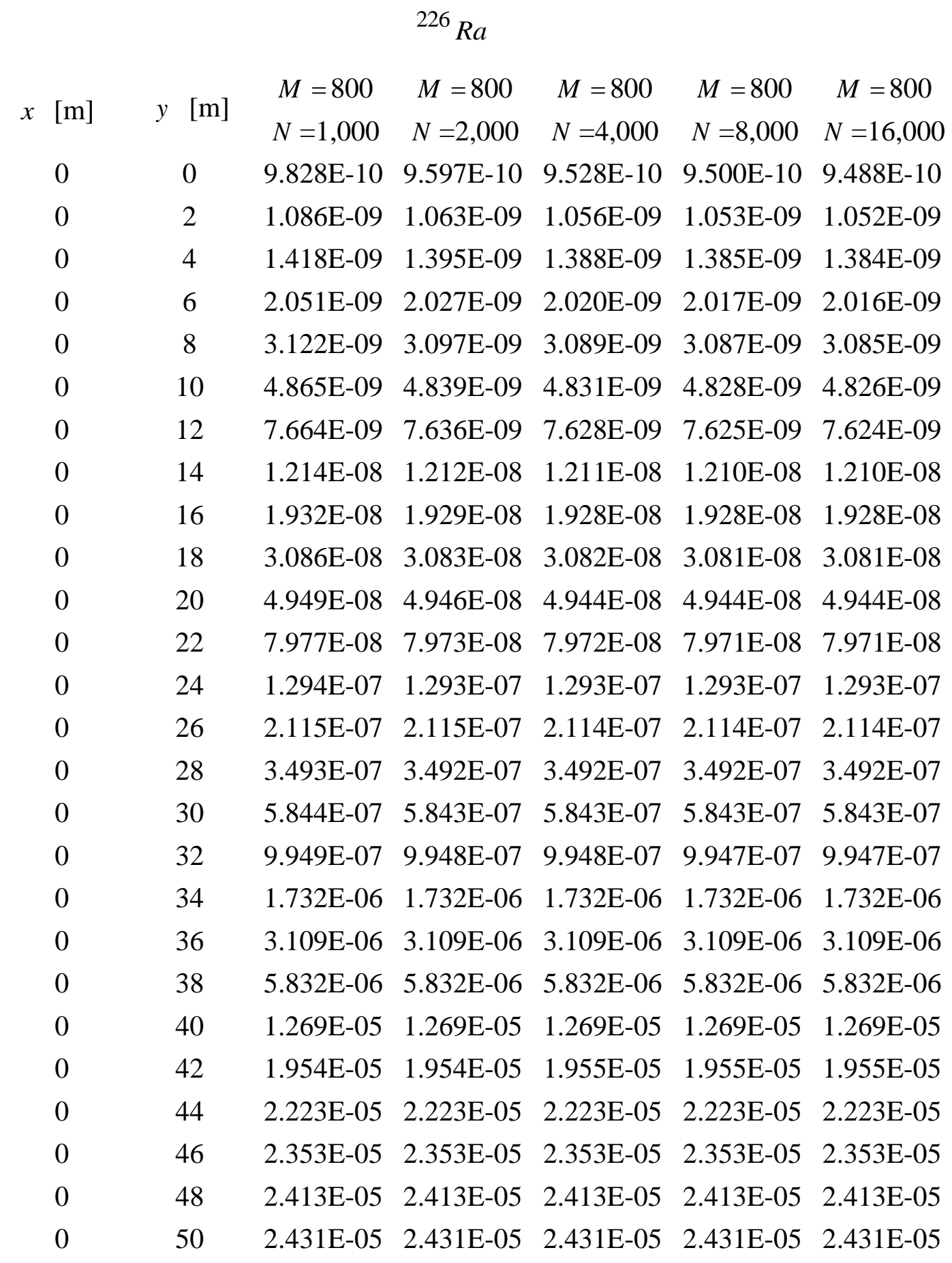




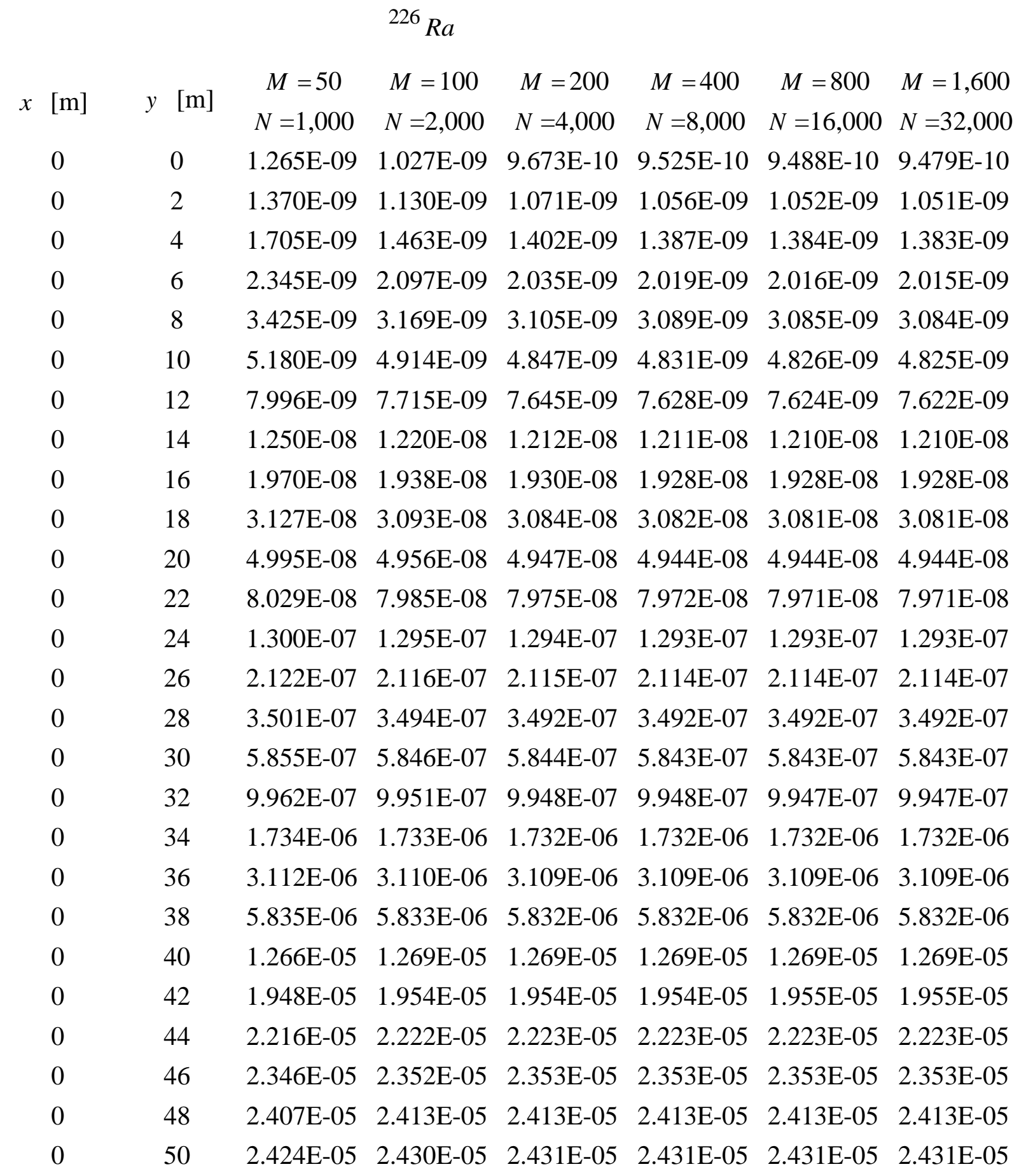




\section{Table S2}

Solution convergence at transect of $x=25 \mathrm{~m}$ for four-species radionuclide transport problem considering simulated domain of $L=250 \mathrm{~m}, W=100 \mathrm{~m}$, subject to Bateman-type sources located at $40 m \leq y \leq 60 m$ for $t=1,000$ year $\quad(M=$ number of terms summed for inverse generalized integral transform; $N=$ number of terms summed for inverse finite Fourier cosine transform).

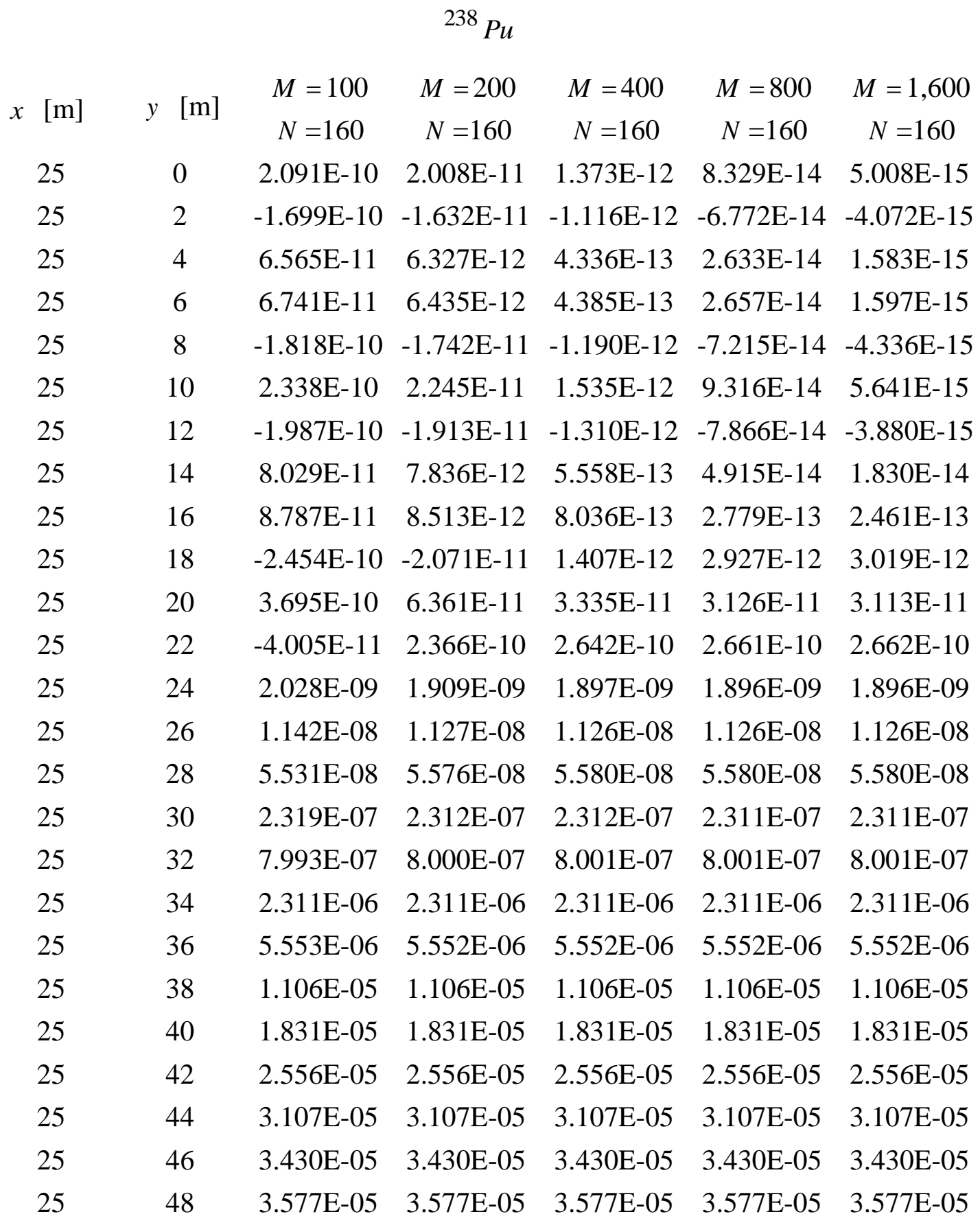




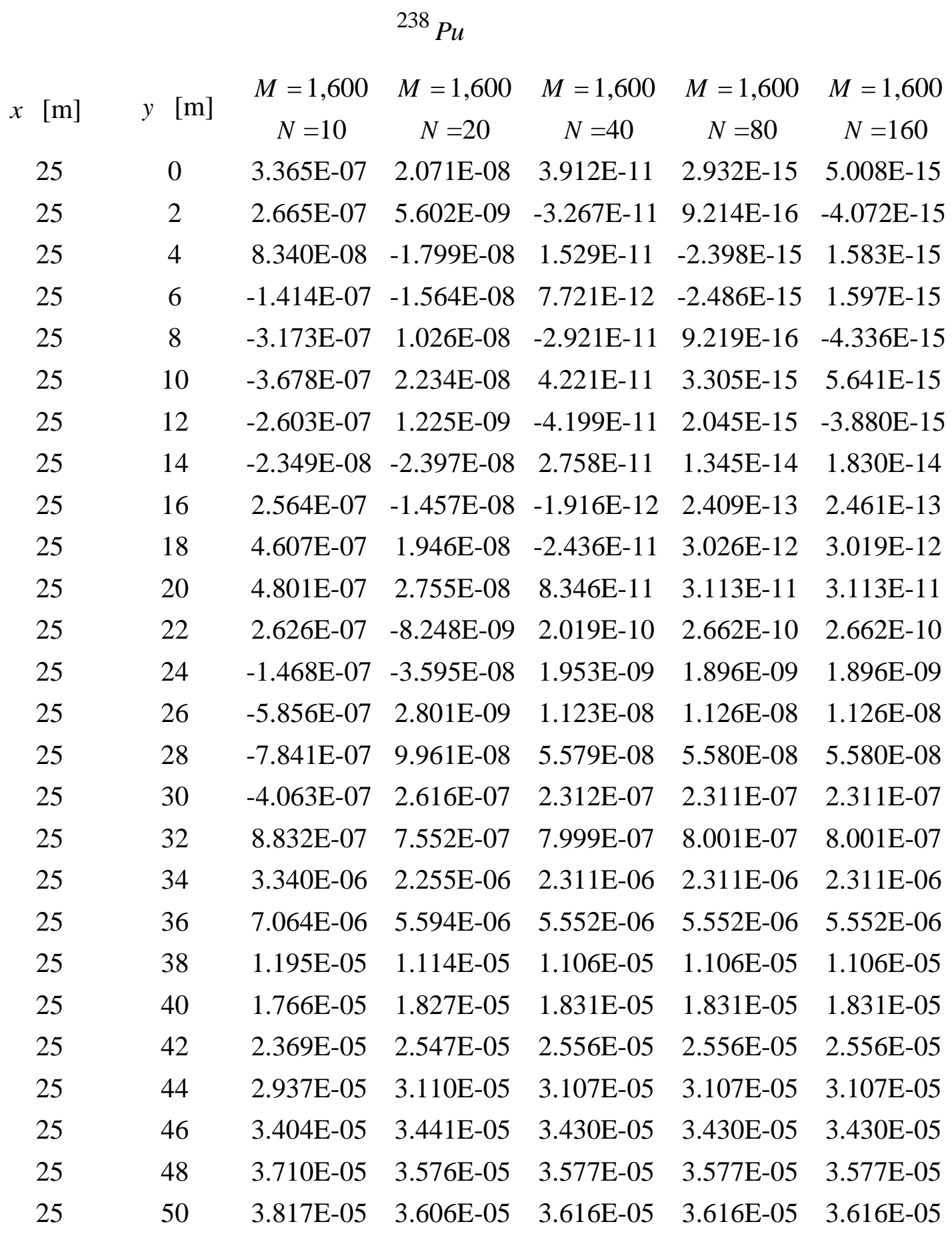




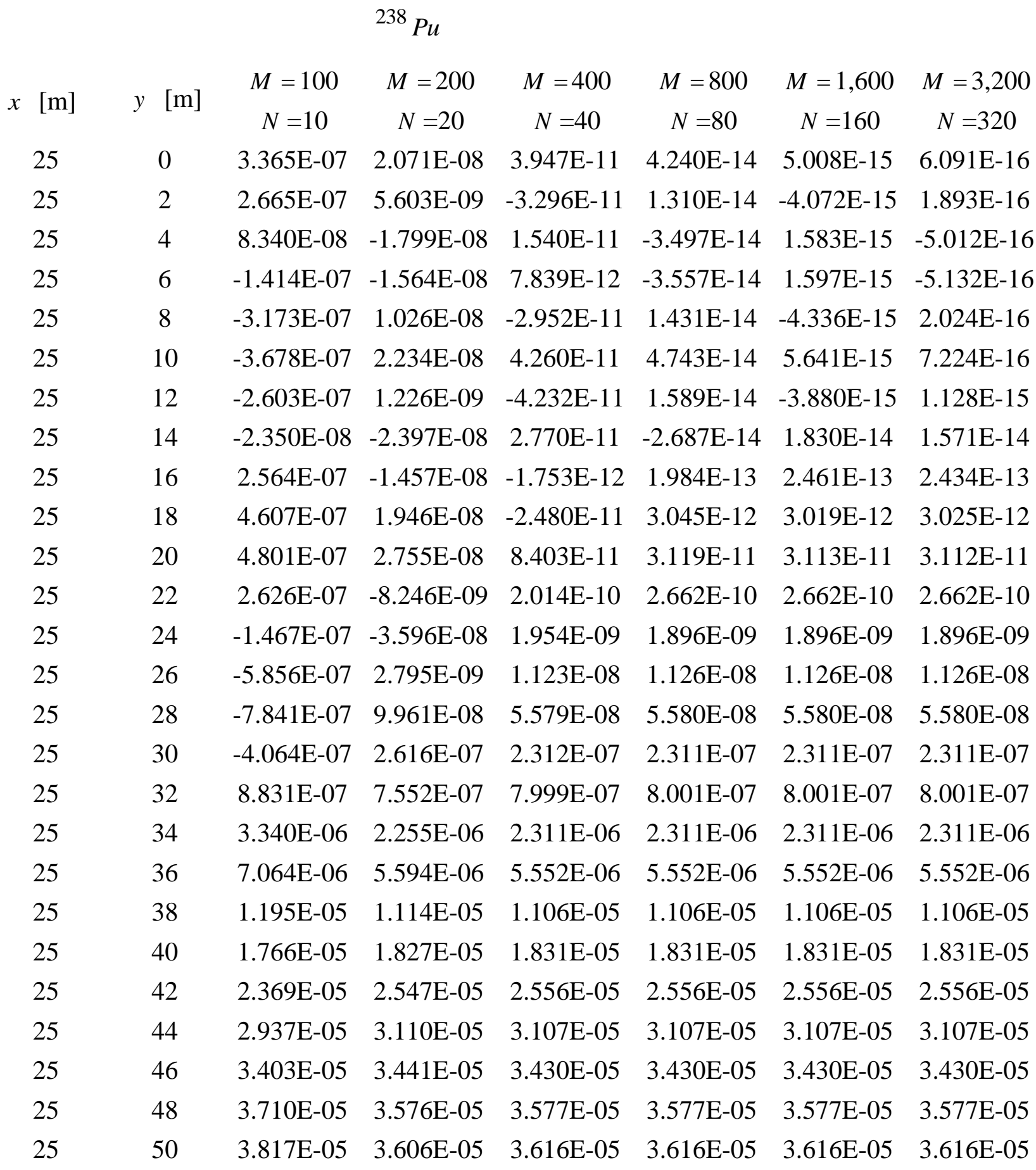




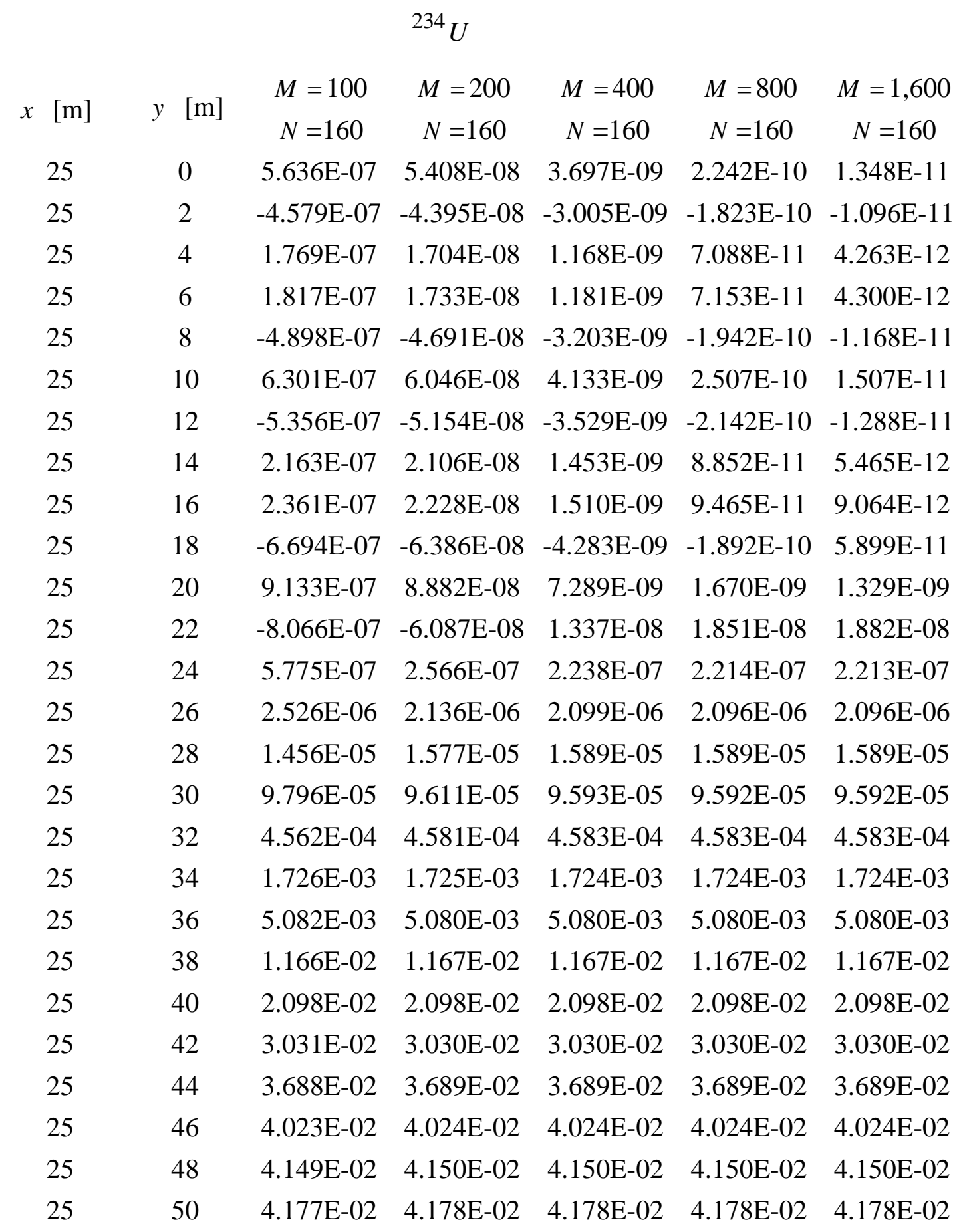




\begin{tabular}{|c|c|c|c|c|c|c|}
\hline$x \quad[\mathrm{~m}]$ & $y \quad[\mathrm{~m}]$ & $\begin{array}{c}M=1,600 \\
N=10\end{array}$ & $\begin{array}{c}M=1,600 \\
N=20\end{array}$ & $\begin{array}{c}M=1,600 \\
N=40\end{array}$ & $\begin{array}{c}M=1,600 \\
N=80\end{array}$ & $\begin{array}{c}M=1,600 \\
N=160\end{array}$ \\
\hline 25 & 0 & 4.634E-04 & 4.349E-05 & $1.190 \mathrm{E}-07$ & 7.944E-12 & $1.348 \mathrm{E}-11$ \\
\hline 25 & 2 & $3.683 \mathrm{E}-04$ & $1.199 \mathrm{E}-05$ & $-9.944 \mathrm{E}-08$ & 2.499E-12 & $-1.096 \mathrm{E}-11$ \\
\hline 25 & 4 & 1.191E-04 & $-3.754 \mathrm{E}-05$ & $4.656 \mathrm{E}-08$ & $-6.494 \mathrm{E}-12$ & 4.263E-12 \\
\hline 25 & 6 & $-1.886 \mathrm{E}-04$ & $-3.339 \mathrm{E}-05$ & $2.346 \mathrm{E}-08$ & $-6.741 \mathrm{E}-12$ & 4.300E-12 \\
\hline 25 & 8 & $-4.329 \mathrm{E}-04$ & $2.068 \mathrm{E}-05$ & $-8.886 \mathrm{E}-08$ & $2.481 \mathrm{E}-12$ & $-1.168 \mathrm{E}-11$ \\
\hline 25 & 10 & $-5.092 \mathrm{E}-04$ & 4.737E-05 & $1.284 \mathrm{E}-07$ & $8.840 \mathrm{E}-12$ & $1.507 \mathrm{E}-11$ \\
\hline 25 & 12 & $-3.709 \mathrm{E}-04$ & 4.289E-06 & $-1.278 \mathrm{E}-07$ & $3.115 \mathrm{E}-12$ & $-1.288 \mathrm{E}-11$ \\
\hline 25 & 14 & $-5.258 \mathrm{E}-05$ & $-5.006 \mathrm{E}-05$ & 8.399E-08 & $-7.624 \mathrm{E}-12$ & $5.465 \mathrm{E}-12$ \\
\hline 25 & 16 & $3.336 \mathrm{E}-04$ & $-3.313 \mathrm{E}-05$ & $-6.736 \mathrm{E}-09$ & $-5.163 \mathrm{E}-12$ & $9.064 \mathrm{E}-12$ \\
\hline 25 & 18 & $6.291 \mathrm{E}-04$ & $3.908 \mathrm{E}-05$ & $-8.310 \mathrm{E}-08$ & 7.794E-11 & $5.899 \mathrm{E}-11$ \\
\hline 25 & 20 & $6.825 \mathrm{E}-04$ & $6.080 \mathrm{E}-05$ & $1.605 \mathrm{E}-07$ & $1.320 \mathrm{E}-09$ & $1.329 \mathrm{E}-09$ \\
\hline 25 & 22 & 4.121E-04 & $-1.361 \mathrm{E}-05$ & $-1.769 \mathrm{E}-07$ & $1.885 \mathrm{E}-08$ & $1.882 \mathrm{E}-08$ \\
\hline 25 & 24 & $-1.414 \mathrm{E}-04$ & -8.207E-05 & $3.968 \mathrm{E}-07$ & 2.213E-07 & 2.213E-07 \\
\hline 25 & 26 & $-7.873 \mathrm{E}-04$ & $-2.350 \mathrm{E}-05$ & $2.001 \mathrm{E}-06$ & 2.096E-06 & $2.096 \mathrm{E}-06$ \\
\hline 25 & 28 & $-1.195 \mathrm{E}-03$ & 1.097E-04 & $1.586 \mathrm{E}-05$ & 1.589E-05 & $1.589 \mathrm{E}-05$ \\
\hline 25 & 30 & $-9.427 \mathrm{E}-04$ & $1.728 \mathrm{E}-04$ & $9.610 \mathrm{E}-05$ & 9.592E-05 & $9.592 \mathrm{E}-05$ \\
\hline 25 & 32 & 3.957E-04 & 3.639E-04 & $4.580 \mathrm{E}-04$ & 4.583E-04 & 4.583E-04 \\
\hline 25 & 34 & $3.154 \mathrm{E}-03$ & $1.588 \mathrm{E}-03$ & $1.725 \mathrm{E}-03$ & $1.724 \mathrm{E}-03$ & $1.724 \mathrm{E}-03$ \\
\hline 25 & 36 & 7.474E-03 & $5.169 \mathrm{E}-03$ & $5.079 \mathrm{E}-03$ & $5.080 \mathrm{E}-03$ & $5.080 \mathrm{E}-03$ \\
\hline 25 & 38 & $1.324 \mathrm{E}-02$ & $1.186 \mathrm{E}-02$ & $1.167 \mathrm{E}-02$ & 1.167E-02 & $1.167 \mathrm{E}-02$ \\
\hline 25 & 40 & 2.007E-02 & $2.090 \mathrm{E}-02$ & $2.098 \mathrm{E}-02$ & 2.098E-02 & 2.098E-02 \\
\hline 25 & 42 & $2.732 \mathrm{E}-02$ & 3.007E-02 & $3.030 \mathrm{E}-02$ & $3.030 \mathrm{E}-02$ & $3.030 \mathrm{E}-02$ \\
\hline 25 & 44 & $3.418 \mathrm{E}-02$ & 3.695E-02 & $3.689 \mathrm{E}-02$ & $3.689 \mathrm{E}-02$ & 3.689E-02 \\
\hline 25 & 46 & 3.984E-02 & 4.049E-02 & $4.024 \mathrm{E}-02$ & 4.024E-02 & 4.024E-02 \\
\hline 25 & 48 & 4.357E-02 & 4.147E-02 & $4.150 \mathrm{E}-02$ & 4.150E-02 & 4.150E-02 \\
\hline 25 & 50 & 4.487E-02 & 4.153E-02 & $4.178 \mathrm{E}-02$ & 4.178E-02 & 4.178E-02 \\
\hline
\end{tabular}




\begin{tabular}{|c|c|c|c|c|c|c|c|}
\hline$x \quad[\mathrm{~m}]$ & $y \quad[\mathrm{~m}]$ & $\begin{array}{c}M=100 \\
N=10\end{array}$ & $\begin{array}{c}M=200 \\
N=20\end{array}$ & $\begin{array}{c}M=400 \\
N=40\end{array}$ & $\begin{array}{c}M=800 \\
N=80\end{array}$ & $\begin{array}{c}M=1,600 \\
N=160\end{array}$ & $\begin{array}{c}M=3,200 \\
N=320\end{array}$ \\
\hline 25 & 0 & 4.633E-04 & $4.350 \mathrm{E}-05$ & 1.200E-07 & $1.137 \mathrm{E}-10$ & $1.348 \mathrm{E}-11$ & $1.642 \mathrm{E}-12$ \\
\hline 25 & 2 & 3.683E-04 & $1.200 \mathrm{E}-05$ & $-1.002 \mathrm{E}-07$ & $3.514 \mathrm{E}-11$ & $-1.096 \mathrm{E}-11$ & $5.103 \mathrm{E}-13$ \\
\hline 25 & 4 & 1.191E-04 & $-3.755 \mathrm{E}-05$ & 4.685E-08 & $-9.378 E-11$ & $4.263 \mathrm{E}-12$ & $-1.351 \mathrm{E}-12$ \\
\hline 25 & 6 & $-1.886 \mathrm{E}-04$ & $-3.340 \mathrm{E}-05$ & 2.377E-08 & $-9.539 \mathrm{E}-11$ & $4.300 \mathrm{E}-12$ & $-1.383 \mathrm{E}-12$ \\
\hline 25 & 8 & $-4.329 \mathrm{E}-04$ & $2.068 \mathrm{E}-05$ & -8.967E-08 & $3.835 \mathrm{E}-11$ & $-1.168 \mathrm{E}-11$ & $5.417 \mathrm{E}-13$ \\
\hline 25 & 10 & $-5.092 \mathrm{E}-04$ & 4.737E-05 & $1.295 \mathrm{E}-07$ & $1.271 \mathrm{E}-10$ & $1.507 \mathrm{E}-11$ & $1.836 \mathrm{E}-12$ \\
\hline 25 & 12 & $-3.709 E-04$ & 4.292E-06 & -1.287E-07 & 4.019E-11 & $-1.288 \mathrm{E}-11$ & $6.071 \mathrm{E}-13$ \\
\hline 25 & 14 & $-5.260 \mathrm{E}-05$ & $-5.007 \mathrm{E}-05$ & 8.432E-08 & $-1.157 \mathrm{E}-10$ & $5.465 \mathrm{E}-12$ & $-1.510 \mathrm{E}-12$ \\
\hline 25 & 16 & 3.336E-04 & $-3.314 \mathrm{E}-05$ & $-6.305 E-09$ & $-1.189 \mathrm{E}-10$ & $9.064 \mathrm{E}-12$ & $1.803 \mathrm{E}-12$ \\
\hline 25 & 18 & $6.290 \mathrm{E}-04$ & 3.908E-05 & $-8.424 \mathrm{E}-08$ & $1.286 \mathrm{E}-10$ & $5.899 \mathrm{E}-11$ & $7.560 \mathrm{E}-11$ \\
\hline 25 & 20 & $6.825 \mathrm{E}-04$ & $6.081 \mathrm{E}-05$ & 1.620E-07 & 1.491E-09 & $1.329 \mathrm{E}-09$ & $1.310 \mathrm{E}-09$ \\
\hline 25 & 22 & 4.121E-04 & $-1.360 \mathrm{E}-05$ & $-1.782 \mathrm{E}-07$ & 1.890E-08 & $1.882 \mathrm{E}-08$ & $1.884 \mathrm{E}-08$ \\
\hline 25 & 24 & $-1.413 \mathrm{E}-04$ & $-8.208 E-05$ & 3.973E-07 & $2.211 \mathrm{E}-07$ & $2.213 \mathrm{E}-07$ & 2.213E-07 \\
\hline 25 & 26 & $-7.871 \mathrm{E}-04$ & $-2.352 \mathrm{E}-05$ & 2.002E-06 & 2.096E-06 & $2.096 \mathrm{E}-06$ & $2.096 \mathrm{E}-06$ \\
\hline 25 & 28 & $-1.194 \mathrm{E}-03$ & $1.097 \mathrm{E}-04$ & $1.586 \mathrm{E}-05$ & 1.589E-05 & $1.589 \mathrm{E}-05$ & $1.589 \mathrm{E}-05$ \\
\hline 25 & 30 & $-9.427 \mathrm{E}-04$ & 1.728E-04 & $9.611 \mathrm{E}-05$ & 9.592E-05 & $9.592 \mathrm{E}-05$ & $9.592 \mathrm{E}-05$ \\
\hline 25 & 32 & $3.955 \mathrm{E}-04$ & 3.639E-04 & $4.580 \mathrm{E}-04$ & 4.583E-04 & $4.583 \mathrm{E}-04$ & $4.583 \mathrm{E}-04$ \\
\hline 25 & 34 & $3.153 \mathrm{E}-03$ & $1.588 \mathrm{E}-03$ & $1.725 \mathrm{E}-03$ & $1.724 \mathrm{E}-03$ & $1.724 \mathrm{E}-03$ & $1.724 \mathrm{E}-03$ \\
\hline 25 & 36 & 7.473E-03 & $5.169 \mathrm{E}-03$ & 5.079E-03 & $5.080 \mathrm{E}-03$ & $5.080 \mathrm{E}-03$ & $5.080 \mathrm{E}-03$ \\
\hline 25 & 38 & 1.324E-02 & $1.186 \mathrm{E}-02$ & 1.167E-02 & 1.167E-02 & $1.167 \mathrm{E}-02$ & $1.167 \mathrm{E}-02$ \\
\hline 25 & 40 & $2.007 \mathrm{E}-02$ & $2.090 \mathrm{E}-02$ & $2.098 \mathrm{E}-02$ & $2.098 \mathrm{E}-02$ & $2.098 \mathrm{E}-02$ & 2.098E-02 \\
\hline 25 & 42 & 2.731E-02 & $3.007 \mathrm{E}-02$ & 3.030E-02 & 3.030E-02 & 3.030E-02 & $3.030 \mathrm{E}-02$ \\
\hline 25 & 44 & $3.418 \mathrm{E}-02$ & $3.695 \mathrm{E}-02$ & 3.689E-02 & 3.689E-02 & $3.689 \mathrm{E}-02$ & $3.689 \mathrm{E}-02$ \\
\hline 25 & 46 & 3.984E-02 & $4.049 \mathrm{E}-02$ & 4.024E-02 & 4.024E-02 & 4.024E-02 & 4.024E-02 \\
\hline 25 & 48 & 4.357E-02 & 4.147E-02 & 4.150E-02 & 4.150E-02 & 4.150E-02 & 4.150E-02 \\
\hline 25 & 50 & 4.487E-02 & $4.153 \mathrm{E}-02$ & 4.178E-02 & 4.178E-02 & $4.178 \mathrm{E}-02$ & 4.178E-02 \\
\hline
\end{tabular}




\begin{tabular}{|c|c|c|c|c|c|c|}
\hline \multirow{3}{*}{$x \quad[\mathrm{~m}]$} & \multicolumn{4}{|c|}{${ }^{230} T h$} & \multirow{3}{*}{$\begin{array}{c}M=800 \\
N=160\end{array}$} & \multirow{3}{*}{$\begin{array}{c}M=1,600 \\
N=160\end{array}$} \\
\hline & & $M=100$ & $M=200$ & $M=400$ & & \\
\hline & & $N=160$ & $N=160$ & $N=160$ & & \\
\hline 25 & 0 & 2.048E-09 & $1.967 \mathrm{E}-10$ & $1.346 \mathrm{E}-11$ & $8.165 \mathrm{E}-13$ & $4.916 \mathrm{E}-14$ \\
\hline 25 & 2 & $-1.664 \mathrm{E}-09$ & $-1.599 \mathrm{E}-10$ & $-1.094 \mathrm{E}-11$ & $-6.637 E-13$ & $-3.985 \mathrm{E}-14$ \\
\hline 25 & 4 & $6.429 \mathrm{E}-10$ & $6.199 \mathrm{E}-11$ & $4.250 \mathrm{E}-12$ & $2.581 \mathrm{E}-13$ & $1.557 \mathrm{E}-14$ \\
\hline 25 & 6 & $6.602 \mathrm{E}-10$ & $6.305 \mathrm{E}-11$ & $4.298 \mathrm{E}-12$ & $2.605 \mathrm{E}-13$ & $1.568 \mathrm{E}-14$ \\
\hline 25 & 8 & $-1.780 \mathrm{E}-09$ & $-1.707 \mathrm{E}-10$ & $-1.166 \mathrm{E}-11$ & $-7.072 \mathrm{E}-13$ & $-4.252 \mathrm{E}-14$ \\
\hline 25 & 10 & $2.290 \mathrm{E}-09$ & $2.199 \mathrm{E}-10$ & $1.505 \mathrm{E}-11$ & $9.128 \mathrm{E}-13$ & $5.489 \mathrm{E}-14$ \\
\hline 25 & 12 & $-1.946 \mathrm{E}-09$ & $-1.875 \mathrm{E}-10$ & $-1.285 \mathrm{E}-11$ & $-7.799 \mathrm{E}-13$ & $-4.690 \mathrm{E}-14$ \\
\hline 25 & 14 & $7.861 \mathrm{E}-10$ & 7.662E-11 & $5.287 \mathrm{E}-12$ & $3.217 \mathrm{E}-13$ & $1.937 \mathrm{E}-14$ \\
\hline 25 & 16 & $8.581 \mathrm{E}-10$ & 8.103E-11 & $5.485 \mathrm{E}-12$ & $3.318 \mathrm{E}-13$ & $2.011 \mathrm{E}-14$ \\
\hline 25 & 18 & $-2.433 \mathrm{E}-09$ & $-2.326 \mathrm{E}-10$ & $-1.586 \mathrm{E}-11$ & $-9.571 \mathrm{E}-13$ & $-5.334 \mathrm{E}-14$ \\
\hline 25 & 20 & 3.314E-09 & $3.184 \mathrm{E}-10$ & $2.186 \mathrm{E}-11$ & $1.408 \mathrm{E}-12$ & $1.668 \mathrm{E}-13$ \\
\hline 25 & 22 & $-2.998 \mathrm{E}-09$ & $-2.885 \mathrm{E}-10$ & $-1.849 \mathrm{E}-11$ & $2.151 \mathrm{E}-13$ & $1.352 \mathrm{E}-12$ \\
\hline 25 & 24 & $1.313 \mathrm{E}-09$ & $1.474 \mathrm{E}-10$ & $2.807 \mathrm{E}-11$ & $1.968 \mathrm{E}-11$ & $1.916 \mathrm{E}-11$ \\
\hline 25 & 26 & $1.770 \mathrm{E}-09$ & $3.538 \mathrm{E}-10$ & $2.189 \mathrm{E}-10$ & $2.099 \mathrm{E}-10$ & $2.093 \mathrm{E}-10$ \\
\hline 25 & 28 & $-2.998 E-09$ & 1.387E-09 & $1.816 \mathrm{E}-09$ & $1.846 \mathrm{E}-09$ & $1.847 \mathrm{E}-09$ \\
\hline 25 & 30 & $2.046 \mathrm{E}-08$ & $1.376 \mathrm{E}-08$ & $1.310 \mathrm{E}-08$ & $1.305 \mathrm{E}-08$ & $1.305 \mathrm{E}-08$ \\
\hline 25 & 32 & $6.522 \mathrm{E}-08$ & 7.223E-08 & 7.294E-08 & 7.299E-08 & 7.299E-08 \\
\hline 25 & 34 & $3.234 \mathrm{E}-07$ & 3.198E-07 & $3.194 \mathrm{E}-07$ & $3.193 \mathrm{E}-07$ & 3.193E-07 \\
\hline 25 & 36 & $1.082 \mathrm{E}-06$ & $1.075 \mathrm{E}-06$ & $1.075 \mathrm{E}-06$ & $1.075 \mathrm{E}-06$ & $1.075 \mathrm{E}-06$ \\
\hline 25 & 38 & 2.696E-06 & $2.731 \mathrm{E}-06$ & $2.735 \mathrm{E}-06$ & $2.735 \mathrm{E}-06$ & 2.735E-06 \\
\hline 25 & 40 & $5.229 \mathrm{E}-06$ & $5.236 \mathrm{E}-06$ & $5.237 \mathrm{E}-06$ & $5.237 \mathrm{E}-06$ & $5.237 \mathrm{E}-06$ \\
\hline 25 & 42 & 7.778E-06 & 7.742E-06 & $7.739 \mathrm{E}-06$ & 7.739E-06 & 7.739E-06 \\
\hline 25 & 44 & $9.382 \mathrm{E}-06$ & 9.398E-06 & $9.399 \mathrm{E}-06$ & $9.399 \mathrm{E}-06$ & 9.399E-06 \\
\hline 25 & 46 & $1.014 \mathrm{E}-05$ & $1.015 \mathrm{E}-05$ & $1.015 \mathrm{E}-05$ & $1.015 \mathrm{E}-05$ & $1.015 \mathrm{E}-05$ \\
\hline 25 & 48 & $1.041 \mathrm{E}-05$ & $1.040 \mathrm{E}-05$ & $1.040 \mathrm{E}-05$ & $1.040 \mathrm{E}-05$ & $1.040 \mathrm{E}-05$ \\
\hline 25 & 50 & $1.042 \mathrm{E}-05$ & $1.045 \mathrm{E}-05$ & $1.045 \mathrm{E}-05$ & $1.045 \mathrm{E}-05$ & $1.045 \mathrm{E}-05$ \\
\hline
\end{tabular}




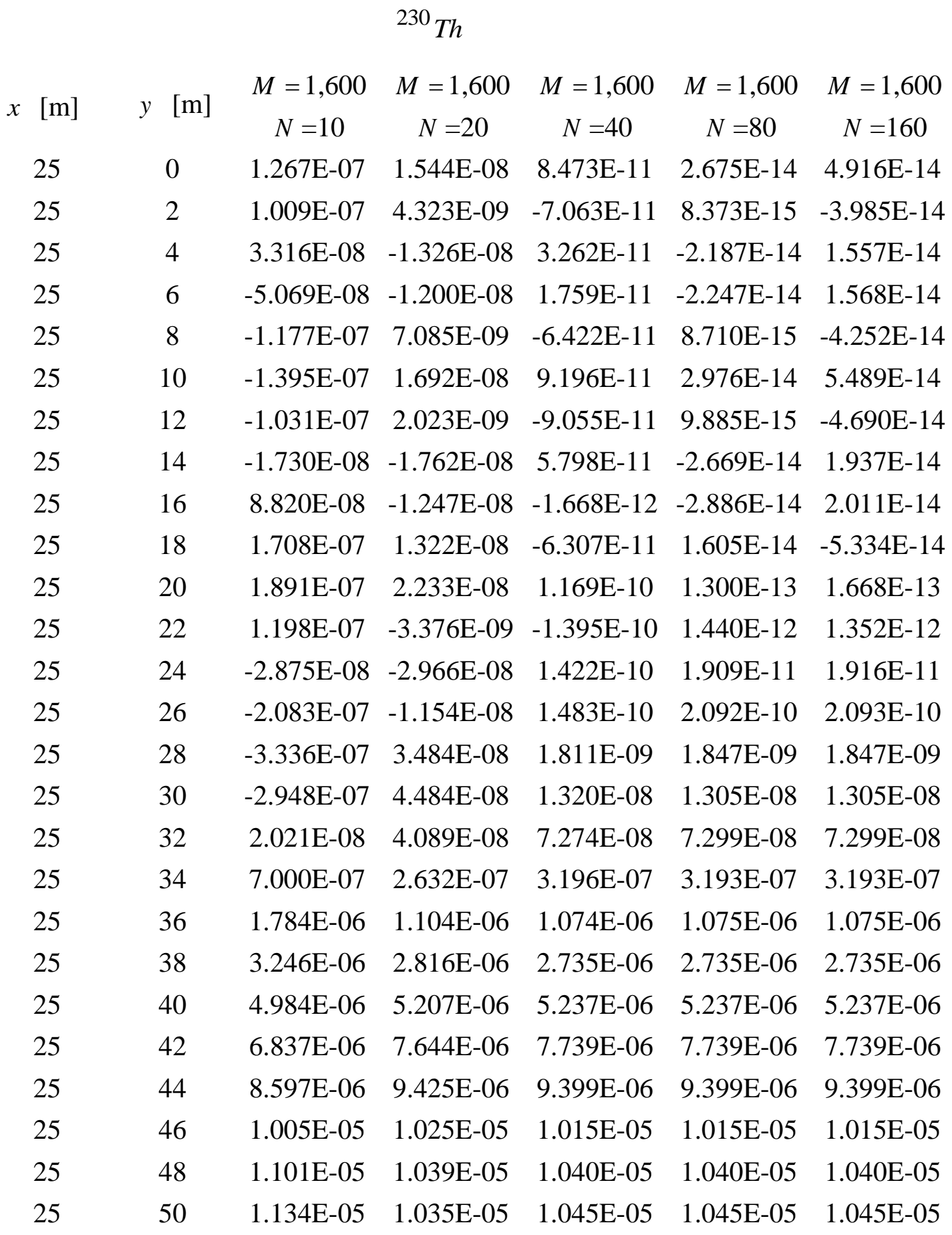




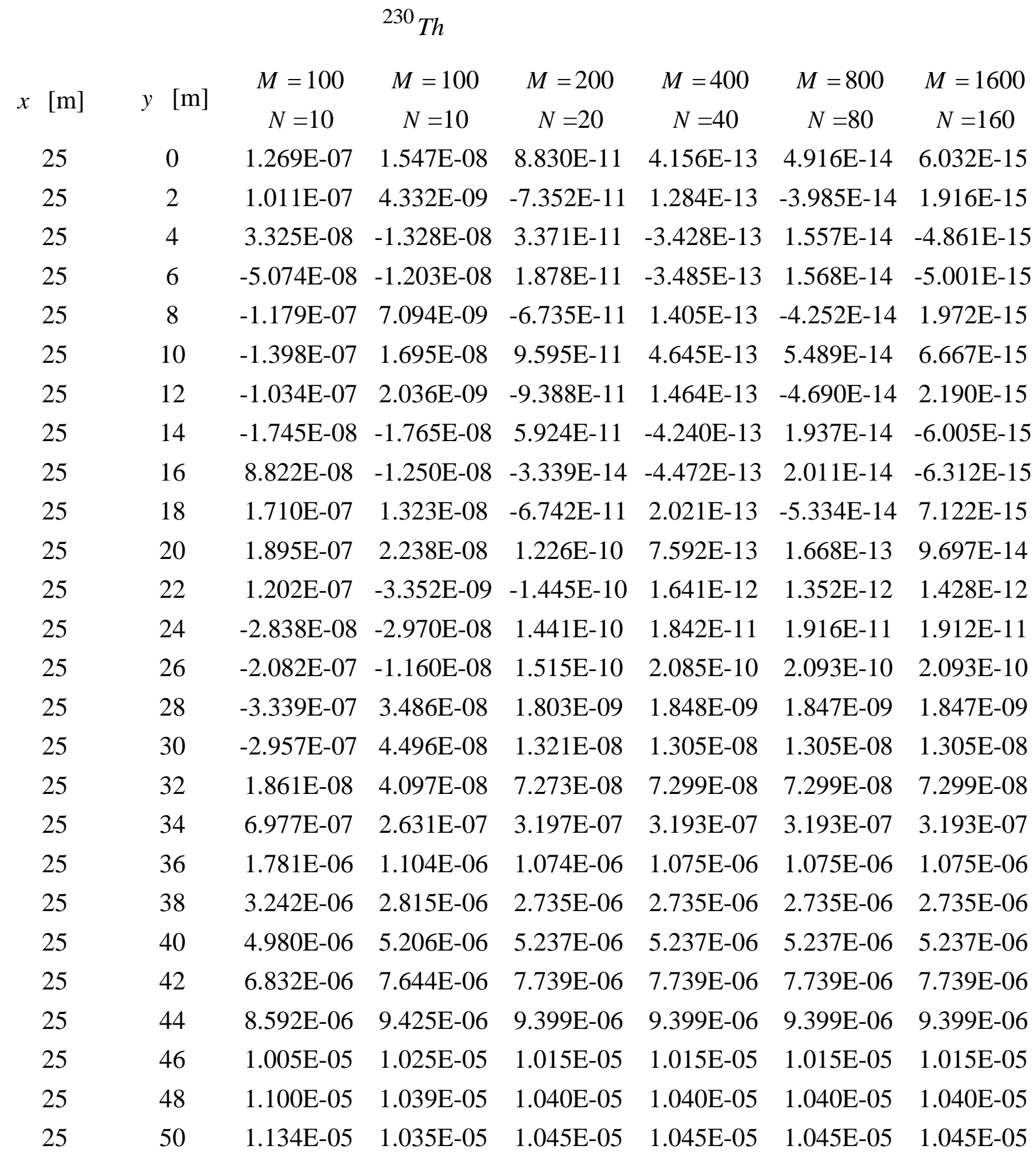




\begin{tabular}{|c|c|c|c|c|c|c|}
\hline \multirow{3}{*}{$x \quad[\mathrm{~m}]$} & \multicolumn{4}{|c|}{${ }^{226} R a$} & \multirow[b]{2}{*}{$M=200$} & \multirow{3}{*}{$\begin{array}{c}M=400 \\
N=160\end{array}$} \\
\hline & & $M=25$ & $M=50$ & $M=100$ & & \\
\hline & & $N=160$ & $N=160$ & $N=160$ & $N=160$ & \\
\hline 25 & 0 & 4.407E-09 & 5.642E-09 & 5.792E-09 & 5.775E-09 & 5.774E-09 \\
\hline 25 & 2 & 7.482E-09 & $6.480 \mathrm{E}-09$ & 6.358E-09 & 6.372E-09 & 6.373E-09 \\
\hline 25 & 4 & 7.864E-09 & $8.251 \mathrm{E}-09$ & 8.298E-09 & 8.292E-09 & 8.292E-09 \\
\hline 25 & 6 & 1.147E-08 & 1.187E-08 & 1.192E-08 & $1.192 \mathrm{E}-08$ & 1.191E-08 \\
\hline 25 & 8 & $1.915 \mathrm{E}-08$ & $1.808 \mathrm{E}-08$ & $1.795 \mathrm{E}-08$ & $1.796 \mathrm{E}-08$ & $1.797 \mathrm{E}-08$ \\
\hline 25 & 10 & 2.613E-08 & $2.751 \mathrm{E}-08$ & 2.767E-08 & $2.765 \mathrm{E}-08$ & $2.765 \mathrm{E}-08$ \\
\hline 25 & 12 & 4.420E-08 & 4.303E-08 & 4.288E-08 & 4.290E-08 & 4.290E-08 \\
\hline 25 & 14 & 6.622E-08 & 6.669E-08 & $6.675 \mathrm{E}-08$ & $6.674 \mathrm{E}-08$ & $6.674 \mathrm{E}-08$ \\
\hline 25 & 16 & $1.033 \mathrm{E}-07$ & $1.038 \mathrm{E}-07$ & 1.039E-07 & 1.039E-07 & $1.039 \mathrm{E}-07$ \\
\hline 25 & 18 & $1.633 \mathrm{E}-07$ & $1.618 \mathrm{E}-07$ & $1.616 \mathrm{E}-07$ & $1.617 \mathrm{E}-07$ & $1.617 \mathrm{E}-07$ \\
\hline 25 & 20 & $2.492 \mathrm{E}-07$ & $2.512 \mathrm{E}-07$ & $2.514 \mathrm{E}-07$ & $2.514 \mathrm{E}-07$ & $2.514 \mathrm{E}-07$ \\
\hline 25 & 22 & $3.925 \mathrm{E}-07$ & $3.907 \mathrm{E}-07$ & $3.905 \mathrm{E}-07$ & $3.905 \mathrm{E}-07$ & $3.905 \mathrm{E}-07$ \\
\hline 25 & 24 & $6.048 \mathrm{E}-07$ & $6.055 \mathrm{E}-07$ & $6.056 \mathrm{E}-07$ & $6.056 \mathrm{E}-07$ & $6.056 \mathrm{E}-07$ \\
\hline 25 & 26 & $9.360 \mathrm{E}-07$ & $9.370 \mathrm{E}-07$ & $9.371 \mathrm{E}-07$ & $9.371 \mathrm{E}-07$ & $9.371 \mathrm{E}-07$ \\
\hline 25 & 28 & $1.448 \mathrm{E}-06$ & $1.445 \mathrm{E}-06$ & $1.444 \mathrm{E}-06$ & $1.444 \mathrm{E}-06$ & $1.444 \mathrm{E}-06$ \\
\hline 25 & 30 & $2.208 \mathrm{E}-06$ & $2.212 \mathrm{E}-06$ & 2.213E-06 & $2.213 \mathrm{E}-06$ & $2.213 \mathrm{E}-06$ \\
\hline 25 & 32 & $3.360 \mathrm{E}-06$ & $3.355 \mathrm{E}-06$ & $3.355 \mathrm{E}-06$ & 3.355E-06 & $3.355 \mathrm{E}-06$ \\
\hline 25 & 34 & $5.001 \mathrm{E}-06$ & $5.002 \mathrm{E}-06$ & $5.002 \mathrm{E}-06$ & $5.002 \mathrm{E}-06$ & $5.002 \mathrm{E}-06$ \\
\hline 25 & 36 & $7.268 \mathrm{E}-06$ & 7.262E-06 & 7.262E-06 & $7.262 \mathrm{E}-06$ & 7.262E-06 \\
\hline 25 & 38 & $1.025 \mathrm{E}-05$ & $1.013 \mathrm{E}-05$ & $1.013 \mathrm{E}-05$ & $1.013 \mathrm{E}-05$ & $1.013 \mathrm{E}-05$ \\
\hline 25 & 40 & $1.356 \mathrm{E}-05$ & $1.338 \mathrm{E}-05$ & $1.337 \mathrm{E}-05$ & $1.337 \mathrm{E}-05$ & $1.337 \mathrm{E}-05$ \\
\hline 25 & 42 & $1.681 \mathrm{E}-05$ & $1.657 \mathrm{E}-05$ & $1.656 \mathrm{E}-05$ & $1.656 \mathrm{E}-05$ & $1.656 \mathrm{E}-05$ \\
\hline 25 & 44 & $1.964 \mathrm{E}-05$ & $1.928 \mathrm{E}-05$ & $1.927 \mathrm{E}-05$ & $1.927 \mathrm{E}-05$ & $1.927 \mathrm{E}-05$ \\
\hline 25 & 46 & $2.161 \mathrm{E}-05$ & $2.125 \mathrm{E}-05$ & $2.123 \mathrm{E}-05$ & $2.123 \mathrm{E}-05$ & $2.123 \mathrm{E}-05$ \\
\hline 25 & 48 & $2.276 \mathrm{E}-05$ & $2.240 \mathrm{E}-05$ & 2.239E-05 & $2.239 \mathrm{E}-05$ & 2.239E-05 \\
\hline 25 & 50 & $2.316 \mathrm{E}-05$ & $2.278 \mathrm{E}-05$ & $2.277 \mathrm{E}-05$ & $2.277 \mathrm{E}-05$ & $2.277 \mathrm{E}-05$ \\
\hline
\end{tabular}




\begin{tabular}{|c|c|c|c|c|c|c|}
\hline & & & & & & \\
\hline$x \quad[\mathrm{~m}]$ & $y \quad[\mathrm{~m}]$ & $\begin{array}{c}M=400 \\
N=10\end{array}$ & $\begin{array}{c}M=400 \\
N=20\end{array}$ & $\begin{array}{c}M=400 \\
N=40\end{array}$ & $\begin{array}{c}M=400 \\
N=80\end{array}$ & $\begin{aligned} M & =400 \\
N & =160\end{aligned}$ \\
\hline 25 & 0 & 8.213E-08 & 8.219E-09 & 5.778E-09 & 5.773E-09 & 5.774E-09 \\
\hline 25 & 2 & $6.616 \mathrm{E}-08$ & 7.029E-09 & 6.369E-09 & $6.373 \mathrm{E}-09$ & 6.373E-09 \\
\hline 25 & 4 & 2.509E-08 & $6.163 \mathrm{E}-09$ & 8.294E-09 & 8.292E-09 & 8.292E-09 \\
\hline 25 & 6 & $-2.302 \mathrm{E}-08$ & $1.008 \mathrm{E}-08$ & $1.192 \mathrm{E}-08$ & 1.191E-08 & $1.191 \mathrm{E}-08$ \\
\hline 25 & 8 & $-5.552 \mathrm{E}-08$ & $1.919 \mathrm{E}-08$ & 1.796E-08 & $1.797 \mathrm{E}-08$ & $1.797 \mathrm{E}-08$ \\
\hline 25 & 10 & $-5.355 \mathrm{E}-08$ & 3.027E-08 & $2.766 \mathrm{E}-08$ & $2.765 \mathrm{E}-08$ & $2.765 \mathrm{E}-08$ \\
\hline 25 & 12 & $-9.356 \mathrm{E}-09$ & 4.301E-08 & 4.290E-08 & 4.290E-08 & 4.290E-08 \\
\hline 25 & 14 & 7.068E-08 & $6.392 \mathrm{E}-08$ & $6.675 \mathrm{E}-08$ & $6.674 \mathrm{E}-08$ & $6.674 \mathrm{E}-08$ \\
\hline 25 & 16 & $1.687 \mathrm{E}-07$ & $1.022 \mathrm{E}-07$ & 1.039E-07 & $1.039 \mathrm{E}-07$ & $1.039 \mathrm{E}-07$ \\
\hline 25 & 18 & 2.642E-07 & $1.640 \mathrm{E}-07$ & $1.617 \mathrm{E}-07$ & $1.617 \mathrm{E}-07$ & $1.617 \mathrm{E}-07$ \\
\hline 25 & 20 & 3.464E-07 & $2.546 \mathrm{E}-07$ & $2.514 \mathrm{E}-07$ & $2.514 \mathrm{E}-07$ & $2.514 \mathrm{E}-07$ \\
\hline 25 & 22 & $4.278 \mathrm{E}-07$ & 3.894E-07 & $3.905 \mathrm{E}-07$ & $3.905 \mathrm{E}-07$ & $3.905 \mathrm{E}-07$ \\
\hline 25 & 24 & $5.536 \mathrm{E}-07$ & $6.012 \mathrm{E}-07$ & $6.056 \mathrm{E}-07$ & $6.056 \mathrm{E}-07$ & $6.056 \mathrm{E}-07$ \\
\hline 25 & 26 & 8.040E-07 & $9.362 \mathrm{E}-07$ & $9.371 \mathrm{E}-07$ & $9.371 \mathrm{E}-07$ & $9.371 \mathrm{E}-07$ \\
\hline 25 & 28 & $1.287 \mathrm{E}-06$ & $1.449 \mathrm{E}-06$ & $1.444 \mathrm{E}-06$ & $1.444 \mathrm{E}-06$ & $1.444 \mathrm{E}-06$ \\
\hline 25 & 30 & $2.120 \mathrm{E}-06$ & $2.216 \mathrm{E}-06$ & 2.213E-06 & 2.213E-06 & $2.213 \mathrm{E}-06$ \\
\hline 25 & 32 & $3.406 \mathrm{E}-06$ & $3.350 \mathrm{E}-06$ & $3.355 \mathrm{E}-06$ & $3.355 \mathrm{E}-06$ & $3.355 \mathrm{E}-06$ \\
\hline 25 & 34 & $5.207 \mathrm{E}-06$ & 4.996E-06 & $5.002 \mathrm{E}-06$ & $5.002 \mathrm{E}-06$ & $5.002 \mathrm{E}-06$ \\
\hline 25 & 36 & 7.517E-06 & 7.267E-06 & 7.262E-06 & 7.262E-06 & 7.262E-06 \\
\hline 25 & 38 & $1.025 \mathrm{E}-05$ & $1.014 \mathrm{E}-05$ & $1.013 \mathrm{E}-05$ & $1.013 \mathrm{E}-05$ & $1.013 \mathrm{E}-05$ \\
\hline 25 & 40 & $1.324 \mathrm{E}-05$ & $1.336 \mathrm{E}-05$ & $1.337 \mathrm{E}-05$ & $1.337 \mathrm{E}-05$ & $1.337 \mathrm{E}-05$ \\
\hline 25 & 42 & $1.624 \mathrm{E}-05$ & $1.655 \mathrm{E}-05$ & $1.656 \mathrm{E}-05$ & $1.656 \mathrm{E}-05$ & $1.656 \mathrm{E}-05$ \\
\hline 25 & 44 & $1.898 \mathrm{E}-05$ & $1.927 \mathrm{E}-05$ & $1.927 \mathrm{E}-05$ & $1.927 \mathrm{E}-05$ & $1.927 \mathrm{E}-05$ \\
\hline 25 & 46 & $2.119 \mathrm{E}-05$ & $2.124 \mathrm{E}-05$ & $2.123 \mathrm{E}-05$ & $2.123 \mathrm{E}-05$ & $2.123 \mathrm{E}-05$ \\
\hline 25 & 48 & $2.262 \mathrm{E}-05$ & $2.239 \mathrm{E}-05$ & $2.239 \mathrm{E}-05$ & 2.239E-05 & $2.239 \mathrm{E}-05$ \\
\hline 25 & 50 & $2.311 \mathrm{E}-05$ & $2.275 \mathrm{E}-05$ & $2.277 \mathrm{E}-05$ & $2.277 \mathrm{E}-05$ & $2.277 \mathrm{E}-05$ \\
\hline
\end{tabular}




\begin{tabular}{|c|c|c|c|c|c|c|c|}
\hline & & & & & & & \\
\hline & & $M=25$ & $M=50$ & $M=100$ & $M=200$ & $M=400$ & $M=800$ \\
\hline & & $N=10$ & $N=20$ & $N=40$ & $N=80$ & $N=160$ & $N=320$ \\
\hline 25 & 0 & 8.713E-08 & $8.228 \mathrm{E}-09$ & 5.787E-09 & 5.774E-09 & 5.774E-09 & 5.773E-09 \\
\hline 25 & 2 & 7.014E-08 & 7.030E-09 & $6.362 \mathrm{E}-09$ & $6.373 \mathrm{E}-09$ & $6.373 \mathrm{E}-09$ & $6.373 \mathrm{E}-09$ \\
\hline 25 & 4 & 2.640E-08 & $6.153 \mathrm{E}-09$ & 8.296E-09 & 8.291E-09 & 8.292E-09 & 8.292E-09 \\
\hline 25 & 6 & $-2.502 \mathrm{E}-08$ & $1.008 \mathrm{E}-08$ & $1.192 \mathrm{E}-08$ & 1.191E-08 & 1.191E-08 & $1.191 \mathrm{E}-08$ \\
\hline 25 & 8 & $-6.016 \mathrm{E}-08$ & $1.921 \mathrm{E}-08$ & $1.795 \mathrm{E}-08$ & $1.797 \mathrm{E}-08$ & $1.797 \mathrm{E}-08$ & $1.797 \mathrm{E}-08$ \\
\hline 25 & 10 & $-5.907 \mathrm{E}-08$ & $3.028 \mathrm{E}-08$ & 2.767E-08 & $2.765 \mathrm{E}-08$ & $2.765 \mathrm{E}-08$ & $2.765 \mathrm{E}-08$ \\
\hline 25 & 12 & $-1.345 \mathrm{E}-08$ & 4.300E-08 & 4.289E-08 & 4.290E-08 & 4.290E-08 & $4.290 \mathrm{E}-08$ \\
\hline 25 & 14 & $6.998 \mathrm{E}-08$ & $6.390 \mathrm{E}-08$ & $6.675 \mathrm{E}-08$ & 6.674E-08 & 6.674E-08 & $6.674 \mathrm{E}-08$ \\
\hline 25 & 16 & $1.722 \mathrm{E}-07$ & $1.022 \mathrm{E}-07$ & 1.039E-07 & $1.039 \mathrm{E}-07$ & 1.039E-07 & $1.039 \mathrm{E}-07$ \\
\hline 25 & 18 & $2.710 \mathrm{E}-07$ & $1.640 \mathrm{E}-07$ & $1.616 \mathrm{E}-07$ & $1.617 \mathrm{E}-07$ & $1.617 \mathrm{E}-07$ & $1.617 \mathrm{E}-07$ \\
\hline 25 & 20 & $3.540 \mathrm{E}-07$ & $2.546 \mathrm{E}-07$ & $2.514 \mathrm{E}-07$ & $2.514 \mathrm{E}-07$ & $2.514 \mathrm{E}-07$ & $2.514 \mathrm{E}-07$ \\
\hline 25 & 22 & 4.327E-07 & 3.894E-07 & $3.905 \mathrm{E}-07$ & $3.905 \mathrm{E}-07$ & $3.905 \mathrm{E}-07$ & $3.905 \mathrm{E}-07$ \\
\hline 25 & 24 & $5.525 \mathrm{E}-07$ & $6.012 \mathrm{E}-07$ & $6.056 \mathrm{E}-07$ & $6.056 \mathrm{E}-07$ & $6.056 \mathrm{E}-07$ & $6.056 \mathrm{E}-07$ \\
\hline 25 & 26 & 7.955E-07 & $9.362 \mathrm{E}-07$ & $9.371 \mathrm{E}-07$ & $9.371 \mathrm{E}-07$ & $9.371 \mathrm{E}-07$ & $9.371 \mathrm{E}-07$ \\
\hline 25 & 28 & $1.273 \mathrm{E}-06$ & $1.450 \mathrm{E}-06$ & $1.444 \mathrm{E}-06$ & $1.444 \mathrm{E}-06$ & $1.444 \mathrm{E}-06$ & $1.444 \mathrm{E}-06$ \\
\hline 25 & 30 & $2.106 \mathrm{E}-06$ & $2.216 \mathrm{E}-06$ & $2.213 \mathrm{E}-06$ & $2.213 \mathrm{E}-06$ & $2.213 \mathrm{E}-06$ & $2.213 \mathrm{E}-06$ \\
\hline 25 & 32 & $3.403 \mathrm{E}-06$ & 3.349E-06 & $3.355 \mathrm{E}-06$ & $3.355 \mathrm{E}-06$ & $3.355 \mathrm{E}-06$ & $3.355 \mathrm{E}-06$ \\
\hline 25 & 34 & $5.228 \mathrm{E}-06$ & 4.996E-06 & $5.002 \mathrm{E}-06$ & $5.002 \mathrm{E}-06$ & $5.002 \mathrm{E}-06$ & $5.002 \mathrm{E}-06$ \\
\hline 25 & 36 & 7.578E-06 & $7.268 \mathrm{E}-06$ & 7.262E-06 & 7.262E-06 & 7.262E-06 & 7.262E-06 \\
\hline 25 & 38 & $1.036 \mathrm{E}-05$ & $1.014 \mathrm{E}-05$ & $1.013 \mathrm{E}-05$ & $1.013 \mathrm{E}-05$ & $1.013 \mathrm{E}-05$ & $1.013 \mathrm{E}-05$ \\
\hline 25 & 40 & $1.342 \mathrm{E}-05$ & $1.337 \mathrm{E}-05$ & $1.337 \mathrm{E}-05$ & $1.337 \mathrm{E}-05$ & $1.337 \mathrm{E}-05$ & $1.337 \mathrm{E}-05$ \\
\hline 25 & 42 & $1.649 \mathrm{E}-05$ & $1.656 \mathrm{E}-05$ & $1.656 \mathrm{E}-05$ & $1.656 \mathrm{E}-05$ & $1.656 \mathrm{E}-05$ & $1.656 \mathrm{E}-05$ \\
\hline 25 & 44 & $1.930 \mathrm{E}-05$ & $1.929 \mathrm{E}-05$ & $1.927 \mathrm{E}-05$ & $1.927 \mathrm{E}-05$ & $1.927 \mathrm{E}-05$ & $1.927 \mathrm{E}-05$ \\
\hline 25 & 46 & $2.156 \mathrm{E}-05$ & $2.126 \mathrm{E}-05$ & $2.123 \mathrm{E}-05$ & $2.123 \mathrm{E}-05$ & $2.123 \mathrm{E}-05$ & $2.123 \mathrm{E}-05$ \\
\hline 25 & 48 & 2.302E-05 & $2.240 \mathrm{E}-05$ & $2.239 \mathrm{E}-05$ & 2.239E-05 & 2.239E-05 & $2.239 \mathrm{E}-05$ \\
\hline 25 & 50 & $2.353 \mathrm{E}-05$ & $2.277 \mathrm{E}-05$ & $2.277 \mathrm{E}-05$ & $2.277 \mathrm{E}-05$ & $2.277 \mathrm{E}-05$ & $2.277 \mathrm{E}-05$ \\
\hline
\end{tabular}




\section{Table S.3}

Solution convergence at transect of exit boundary $(x=250 \mathrm{~m})$ for four-species radionuclide transport problem considering simulated domain of $L=250 \mathrm{~m}$, $W=100 \mathrm{~m}$ subject to Bateman-type sources located at $40 \mathrm{~m} \leq y \leq 60 \mathrm{~m}$ for $t=$ 1000 year $(M=$ number of terms summed for inverse generalized integral transform and $\quad N=$ number of terms summed for inverse finite Fourier cosine transform).

\begin{tabular}{|c|c|c|c|c|c|c|}
\hline$[\mathrm{m}]$ & $y \quad[\mathrm{~m}]$ & $\begin{array}{c}M=400 \\
N=16\end{array}$ & $\begin{array}{c}M=800 \\
N=16\end{array}$ & $\begin{array}{c}M=1,600 \\
N=16\end{array}$ & $\begin{array}{c}M=3,200 \\
N=16\end{array}$ & $\begin{array}{c}M=6,400 \\
N=16\end{array}$ \\
\hline 250 & 0 & $1.897 \mathrm{E}-08$ & $1.759 \mathrm{E}-08$ & $1.751 \mathrm{E}-08$ & $1.750 \mathrm{E}-08$ & $1.750 \mathrm{E}-08$ \\
\hline 250 & 2 & $1.878 \mathrm{E}-08$ & $1.816 \mathrm{E}-08$ & $1.812 \mathrm{E}-08$ & $1.812 \mathrm{E}-08$ & $1.812 \mathrm{E}-08$ \\
\hline 250 & 4 & 1.911E-08 & $1.993 \mathrm{E}-08$ & $1.998 \mathrm{E}-08$ & $1.998 \mathrm{E}-08$ & $1.998 \mathrm{E}-08$ \\
\hline 250 & 6 & $2.166 \mathrm{E}-08$ & 2.304E-08 & $2.313 \mathrm{E}-08$ & $2.314 \mathrm{E}-08$ & $2.314 \mathrm{E}-08$ \\
\hline 250 & 8 & $2.720 \mathrm{E}-08$ & $2.762 \mathrm{E}-08$ & $2.765 \mathrm{E}-08$ & $2.765 \mathrm{E}-08$ & $2.765 \mathrm{E}-08$ \\
\hline 250 & 10 & 3.472E-08 & 3.367E-08 & 3.361E-08 & $3.360 \mathrm{E}-08$ & $3.360 \mathrm{E}-08$ \\
\hline 250 & 12 & 4.260E-08 & 4.119E-08 & $4.110 \mathrm{E}-08$ & $4.110 \mathrm{E}-08$ & $4.110 \mathrm{E}-08$ \\
\hline 250 & 14 & $5.041 \mathrm{E}-08$ & $5.023 \mathrm{E}-08$ & $5.022 \mathrm{E}-08$ & $5.022 \mathrm{E}-08$ & $5.022 \mathrm{E}-08$ \\
\hline 250 & 16 & 5.961E-08 & $6.097 \mathrm{E}-08$ & $6.106 \mathrm{E}-08$ & $6.106 \mathrm{E}-08$ & $6.106 \mathrm{E}-08$ \\
\hline 250 & 18 & 7.213E-08 & 7.358E-08 & 7.367E-08 & 7.367E-08 & 7.367E-08 \\
\hline 250 & 20 & 8.827E-08 & 8.809E-08 & 8.807E-08 & 8.807E-08 & $8.807 \mathrm{E}-08$ \\
\hline 250 & 22 & $1.062 \mathrm{E}-07$ & $1.043 \mathrm{E}-07$ & $1.042 \mathrm{E}-07$ & $1.042 \mathrm{E}-07$ & $1.042 \mathrm{E}-07$ \\
\hline 250 & 24 & $1.235 \mathrm{E}-07$ & $1.221 \mathrm{E}-07$ & $1.220 \mathrm{E}-07$ & $1.220 \mathrm{E}-07$ & $1.220 \mathrm{E}-07$ \\
\hline 250 & 26 & $1.403 \mathrm{E}-07$ & $1.412 \mathrm{E}-07$ & $1.413 \mathrm{E}-07$ & $1.413 \mathrm{E}-07$ & $1.413 \mathrm{E}-07$ \\
\hline 250 & 28 & $1.590 \mathrm{E}-07$ & $1.616 \mathrm{E}-07$ & $1.617 \mathrm{E}-07$ & $1.617 \mathrm{E}-07$ & $1.617 \mathrm{E}-07$ \\
\hline 250 & 30 & $1.820 \mathrm{E}-07$ & $1.830 \mathrm{E}-07$ & $1.831 \mathrm{E}-07$ & $1.831 \mathrm{E}-07$ & $1.831 \mathrm{E}-07$ \\
\hline 250 & 32 & $2.081 \mathrm{E}-07$ & $2.050 \mathrm{E}-07$ & $2.048 \mathrm{E}-07$ & $2.048 \mathrm{E}-07$ & $2.048 \mathrm{E}-07$ \\
\hline 250 & 34 & 2.309E-07 & $2.268 \mathrm{E}-07$ & $2.265 \mathrm{E}-07$ & $2.265 \mathrm{E}-07$ & $2.265 \mathrm{E}-07$ \\
\hline 250 & 36 & $2.424 \mathrm{E}-07$ & $2.472 \mathrm{E}-07$ & $2.475 \mathrm{E}-07$ & $2.475 \mathrm{E}-07$ & $2.475 \mathrm{E}-07$ \\
\hline 250 & 38 & $2.388 \mathrm{E}-07$ & $2.656 \mathrm{E}-07$ & $2.673 \mathrm{E}-07$ & $2.674 \mathrm{E}-07$ & $2.674 \mathrm{E}-07$ \\
\hline 250 & 40 & 2.237E-07 & $2.816 \mathrm{E}-07$ & $2.852 \mathrm{E}-07$ & $2.854 \mathrm{E}-07$ & $2.855 \mathrm{E}-07$ \\
\hline 250 & 42 & $2.070 \mathrm{E}-07$ & $2.953 \mathrm{E}-07$ & $3.008 \mathrm{E}-07$ & $3.011 \mathrm{E}-07$ & $3.011 \mathrm{E}-07$ \\
\hline 250 & 44 & $1.981 \mathrm{E}-07$ & $3.067 \mathrm{E}-07$ & $3.135 \mathrm{E}-07$ & $3.139 \mathrm{E}-07$ & $3.140 \mathrm{E}-07$ \\
\hline 250 & 46 & $1.996 \mathrm{E}-07$ & $3.157 \mathrm{E}-07$ & $3.230 \mathrm{E}-07$ & $3.234 \mathrm{E}-07$ & $3.234 \mathrm{E}-07$ \\
\hline
\end{tabular}




\begin{tabular}{|c|c|c|c|c|c|c|}
\hline 250 & 48 & $2.060 \mathrm{E}-07$ & $3.216 \mathrm{E}-07$ & $3.288 \mathrm{E}-07$ & $3.292 \mathrm{E}-07$ & -07 \\
\hline 250 & 50 & $2.094 \mathrm{E}-07$ & $3.236 \mathrm{E}-07$ & $3.308 \mathrm{E}-07$ & $3.312 \mathrm{E}-07$ & $3.312 \mathrm{E}-07$ \\
\hline \multirow{3}{*}{ [m] } & \multirow{3}{*}{$y \quad[\mathrm{~m}]$} & & ${ }^{226} R a$ & & & \\
\hline & & $M=6,400$ & $M=6,400$ & $M=6,400$ & $M=6,400$ & $M=6,400$ \\
\hline & & $N=1$ & $N=2$ & $N=4$ & $N=8$ & $N=16$ \\
\hline 0 & 0 & $1.529 \mathrm{E}-07$ & $-3.078 \mathrm{E}-09$ & $1.837 \mathrm{E}-08$ & $1.750 \mathrm{E}-08$ & $1.750 \mathrm{E}-08$ \\
\hline 250 & 2 & $1.529 \mathrm{E}-07$ & $-1.848 \mathrm{E}-09$ & $1.892 \mathrm{E}-08$ & $1.812 \mathrm{E}-08$ & $1.812 \mathrm{E}-08$ \\
\hline 250 & 4 & $1.529 \mathrm{E}-07$ & $1.823 \mathrm{E}-09$ & 2.062E-08 & $1.998 \mathrm{E}-08$ & $1.998 \mathrm{E}-08$ \\
\hline 50 & 6 & $1.529 \mathrm{E}-07$ & 7.876E-09 & 2.351E-08 & $2.314 \mathrm{E}-08$ & $2.314 \mathrm{E}-08$ \\
\hline 250 & 8 & $1.529 \mathrm{E}-07$ & $1.622 \mathrm{E}-08$ & 2.771E-08 & $2.765 \mathrm{E}-08$ & $2.765 \mathrm{E}-08$ \\
\hline 250 & 10 & $1.529 \mathrm{E}-07$ & $2.671 \mathrm{E}-08$ & 3.334E-08 & $3.360 \mathrm{E}-08$ & $3.360 \mathrm{E}-08$ \\
\hline 250 & 12 & $1.529 \mathrm{E}-07$ & $3.920 \mathrm{E}-08$ & $4.055 \mathrm{E}-08$ & 4.110E-08 & $4.110 \mathrm{E}-08$ \\
\hline 250 & 14 & $1.529 \mathrm{E}-07$ & $5.348 \mathrm{E}-08$ & 4.946E-08 & $5.022 \mathrm{E}-08$ & $5.022 \mathrm{E}-08$ \\
\hline 250 & 16 & $1.529 \mathrm{E}-07$ & $6.933 \mathrm{E}-08$ & $6.020 \mathrm{E}-08$ & $6.106 \mathrm{E}-08$ & $6.106 \mathrm{E}-08$ \\
\hline 250 & 18 & $1.529 \mathrm{E}-07$ & $8.650 \mathrm{E}-08$ & 7.282E-08 & 7.367E-08 & 7.367E-08 \\
\hline 250 & 20 & $1.529 \mathrm{E}-07$ & $1.047 \mathrm{E}-07$ & $8.736 \mathrm{E}-08$ & 8.807E-08 & 8.807E-08 \\
\hline 250 & 22 & $1.529 \mathrm{E}-07$ & $1.237 \mathrm{E}-07$ & $1.037 \mathrm{E}-07$ & $1.042 \mathrm{E}-07$ & $1.042 \mathrm{E}-07$ \\
\hline 250 & 24 & $1.529 \mathrm{E}-07$ & $1.431 \mathrm{E}-07$ & $1.218 \mathrm{E}-07$ & $1.220 \mathrm{E}-07$ & $1.220 \mathrm{E}-07$ \\
\hline 250 & 26 & $1.529 \mathrm{E}-07$ & $1.627 \mathrm{E}-07$ & $1.414 \mathrm{E}-07$ & $1.413 \mathrm{E}-07$ & $1.413 \mathrm{E}-07$ \\
\hline 250 & 28 & $1.529 \mathrm{E}-07$ & $1.821 \mathrm{E}-07$ & $1.622 \mathrm{E}-07$ & $1.617 \mathrm{E}-07$ & $1.617 \mathrm{E}-07$ \\
\hline 250 & 30 & $1.529 \mathrm{E}-07$ & $2.011 \mathrm{E}-07$ & $1.838 \mathrm{E}-07$ & $1.831 \mathrm{E}-07$ & $1.831 \mathrm{E}-07$ \\
\hline 250 & 32 & $1.529 \mathrm{E}-07$ & $2.193 \mathrm{E}-07$ & 2.057E-07 & $2.048 \mathrm{E}-07$ & $2.048 \mathrm{E}-07$ \\
\hline 250 & 34 & $1.529 \mathrm{E}-07$ & $2.365 \mathrm{E}-07$ & 2.274E-07 & $2.265 \mathrm{E}-07$ & $2.265 \mathrm{E}-07$ \\
\hline 250 & 36 & $1.529 \mathrm{E}-07$ & $2.523 \mathrm{E}-07$ & $2.483 \mathrm{E}-07$ & $2.475 \mathrm{E}-07$ & $2.475 \mathrm{E}-07$ \\
\hline 250 & 38 & $1.529 \mathrm{E}-07$ & $2.666 \mathrm{E}-07$ & $2.680 \mathrm{E}-07$ & 2.674E-07 & $2.674 \mathrm{E}-07$ \\
\hline 250 & 40 & $1.529 \mathrm{E}-07$ & $2.791 \mathrm{E}-07$ & 2.857E-07 & $2.855 \mathrm{E}-07$ & $2.855 \mathrm{E}-07$ \\
\hline 250 & 42 & $1.529 \mathrm{E}-07$ & $2.896 \mathrm{E}-07$ & $3.011 \mathrm{E}-07$ & $3.011 \mathrm{E}-07$ & $3.011 \mathrm{E}-07$ \\
\hline 250 & 44 & $1.529 \mathrm{E}-07$ & $2.979 \mathrm{E}-07$ & $3.136 \mathrm{E}-07$ & $3.140 \mathrm{E}-07$ & $3.140 \mathrm{E}-07$ \\
\hline 250 & 46 & $1.529 \mathrm{E}-07$ & $3.040 \mathrm{E}-07$ & $3.228 \mathrm{E}-07$ & $3.234 \mathrm{E}-07$ & $3.234 \mathrm{E}-07$ \\
\hline 250 & 48 & $1.529 \mathrm{E}-07$ & $3.077 \mathrm{E}-07$ & 3.284E-07 & 3.293E-07 & $3.293 \mathrm{E}-07$ \\
\hline 250 & 50 & $1.529 \mathrm{E}-07$ & $3.089 \mathrm{E}-07$ & 3.303E-07 & $3.312 \mathrm{E}-07$ & $3.312 \mathrm{E}-07$ \\
\hline
\end{tabular}




\begin{tabular}{|c|c|c|c|c|c|c|c|}
\hline$x \quad[\mathrm{~m}]$ & $y \quad[\mathrm{~m}]$ & $\begin{array}{c}M=400 \\
N=1\end{array}$ & $\begin{array}{c}M=800 \\
N=2\end{array}$ & $\begin{array}{c}M=1,600 \\
N=4\end{array}$ & $\begin{array}{c}M=3,200 \\
N=8\end{array}$ & $\begin{array}{c}M=6,400 \\
N=16\end{array}$ & $\begin{array}{c}M= \\
12,800 \\
N=32\end{array}$ \\
\hline 250 & 0 & $1.286 \mathrm{E}-07$ & -1.779E-09 & $1.831 \mathrm{E}-08$ & $1.750 \mathrm{E}-08$ & $1.750 \mathrm{E}-08$ & $1.750 \mathrm{E}-08$ \\
\hline 250 & 2 & $1.286 \mathrm{E}-07$ & $-5.717 \mathrm{E}-10$ & $1.887 \mathrm{E}-08$ & $1.812 \mathrm{E}-08$ & $1.812 \mathrm{E}-08$ & $1.812 \mathrm{E}-08$ \\
\hline 250 & 4 & $1.286 \mathrm{E}-07$ & 3.033E-09 & $2.057 \mathrm{E}-08$ & $1.998 \mathrm{E}-08$ & $1.998 \mathrm{E}-08$ & $1.998 \mathrm{E}-08$ \\
\hline 250 & 6 & $1.286 \mathrm{E}-07$ & 8.977E-09 & $2.348 \mathrm{E}-08$ & $2.314 \mathrm{E}-08$ & $2.314 \mathrm{E}-08$ & $2.314 \mathrm{E}-08$ \\
\hline 250 & 8 & $1.286 \mathrm{E}-07$ & 1.717E-08 & $2.769 \mathrm{E}-08$ & $2.765 \mathrm{E}-08$ & $2.765 \mathrm{E}-08$ & $2.765 \mathrm{E}-08$ \\
\hline 250 & 10 & $1.286 \mathrm{E}-07$ & 2.747E-08 & 3.335E-08 & $3.360 \mathrm{E}-08$ & $3.360 \mathrm{E}-08$ & $3.360 \mathrm{E}-08$ \\
\hline 250 & 12 & $1.286 \mathrm{E}-07$ & 3.974E-08 & 4.057E-08 & $4.110 \mathrm{E}-08$ & $4.110 \mathrm{E}-08$ & 4.110E-08 \\
\hline 250 & 14 & $1.286 \mathrm{E}-07$ & $5.376 \mathrm{E}-08$ & 4.951E-08 & $5.022 \mathrm{E}-08$ & $5.022 \mathrm{E}-08$ & $5.022 \mathrm{E}-08$ \\
\hline 250 & 16 & $1.286 \mathrm{E}-07$ & $6.932 \mathrm{E}-08$ & $6.026 \mathrm{E}-08$ & $6.106 \mathrm{E}-08$ & $6.106 \mathrm{E}-08$ & $6.106 \mathrm{E}-08$ \\
\hline 250 & 18 & $1.286 \mathrm{E}-07$ & 8.618E-08 & $7.289 \mathrm{E}-08$ & 7.367E-08 & 7.367E-08 & 7.367E-08 \\
\hline 250 & 20 & $1.286 \mathrm{E}-07$ & $1.041 \mathrm{E}-07$ & 8.743E-08 & 8.807E-08 & 8.807E-08 & 8.807E-08 \\
\hline 250 & 22 & $1.286 \mathrm{E}-07$ & $1.227 \mathrm{E}-07$ & $1.038 \mathrm{E}-07$ & $1.042 \mathrm{E}-07$ & 1.042E-07 & $1.042 \mathrm{E}-07$ \\
\hline 250 & 24 & $1.286 \mathrm{E}-07$ & $1.418 \mathrm{E}-07$ & $1.219 \mathrm{E}-07$ & $1.220 \mathrm{E}-07$ & $1.220 \mathrm{E}-07$ & $1.220 \mathrm{E}-07$ \\
\hline 250 & 26 & $1.286 \mathrm{E}-07$ & $1.610 \mathrm{E}-07$ & $1.415 \mathrm{E}-07$ & $1.413 \mathrm{E}-07$ & $1.413 \mathrm{E}-07$ & $1.413 \mathrm{E}-07$ \\
\hline 250 & 28 & $1.286 \mathrm{E}-07$ & $1.801 \mathrm{E}-07$ & $1.622 \mathrm{E}-07$ & $1.617 \mathrm{E}-07$ & $1.617 \mathrm{E}-07$ & $1.617 \mathrm{E}-07$ \\
\hline 250 & 30 & $1.286 \mathrm{E}-07$ & $1.987 \mathrm{E}-07$ & $1.837 \mathrm{E}-07$ & $1.831 \mathrm{E}-07$ & $1.831 \mathrm{E}-07$ & $1.831 \mathrm{E}-07$ \\
\hline 250 & 32 & $1.286 \mathrm{E}-07$ & $2.166 \mathrm{E}-07$ & $2.056 \mathrm{E}-07$ & $2.048 \mathrm{E}-07$ & $2.048 \mathrm{E}-07$ & $2.048 \mathrm{E}-07$ \\
\hline 250 & 34 & $1.286 \mathrm{E}-07$ & $2.335 \mathrm{E}-07$ & $2.272 \mathrm{E}-07$ & $2.265 \mathrm{E}-07$ & $2.265 \mathrm{E}-07$ & $2.265 \mathrm{E}-07$ \\
\hline 250 & 36 & $1.286 \mathrm{E}-07$ & $2.490 \mathrm{E}-07$ & $2.481 \mathrm{E}-07$ & $2.475 \mathrm{E}-07$ & $2.475 \mathrm{E}-07$ & $2.475 \mathrm{E}-07$ \\
\hline 250 & 38 & $1.286 \mathrm{E}-07$ & $2.631 \mathrm{E}-07$ & $2.677 \mathrm{E}-07$ & $2.674 \mathrm{E}-07$ & $2.674 \mathrm{E}-07$ & $2.674 \mathrm{E}-07$ \\
\hline 250 & 40 & $1.286 \mathrm{E}-07$ & $2.753 \mathrm{E}-07$ & $2.855 \mathrm{E}-07$ & $2.854 \mathrm{E}-07$ & $2.855 \mathrm{E}-07$ & $2.855 \mathrm{E}-07$ \\
\hline 250 & 42 & $1.286 \mathrm{E}-07$ & $2.856 \mathrm{E}-07$ & $3.008 \mathrm{E}-07$ & $3.011 \mathrm{E}-07$ & $3.011 \mathrm{E}-07$ & $3.012 \mathrm{E}-07$ \\
\hline 250 & 44 & $1.286 \mathrm{E}-07$ & 2.938E-07 & $3.132 \mathrm{E}-07$ & $3.139 \mathrm{E}-07$ & $3.140 \mathrm{E}-07$ & $3.140 \mathrm{E}-07$ \\
\hline 250 & 46 & $1.286 \mathrm{E}-07$ & $2.998 \mathrm{E}-07$ & $3.224 \mathrm{E}-07$ & $3.234 \mathrm{E}-07$ & $3.234 \mathrm{E}-07$ & $3.234 \mathrm{E}-07$ \\
\hline 250 & 48 & $1.286 \mathrm{E}-07$ & $3.034 \mathrm{E}-07$ & $3.280 \mathrm{E}-07$ & $3.292 \mathrm{E}-07$ & $3.293 \mathrm{E}-07$ & $3.293 \mathrm{E}-07$ \\
\hline 250 & 50 & $1.286 \mathrm{E}-07$ & $3.046 \mathrm{E}-07$ & $3.299 \mathrm{E}-07$ & $3.312 \mathrm{E}-07$ & $3.312 \mathrm{E}-07$ & $3.312 \mathrm{E}-07$ \\
\hline
\end{tabular}




\section{Table S4}

Solution convergence at transect of inlet boundary $\left(\begin{array}{ccc}x=0 & \mathrm{~m}\end{array}\right)$ for four-species radionuclide transport problem considering simulated domain of $L=2,500 \mathrm{~m}$, $W=100 \mathrm{~m}$ subject to Bateman-type sources located at $45 \mathrm{~m} \leq y \leq 55 \mathrm{~m}$ for $t=$ 1,000 year $\quad(M=$ number of terms summed for inverse generalized integral transform; $N=$ number of terms summed for inverse finite Fourier cosine transform).

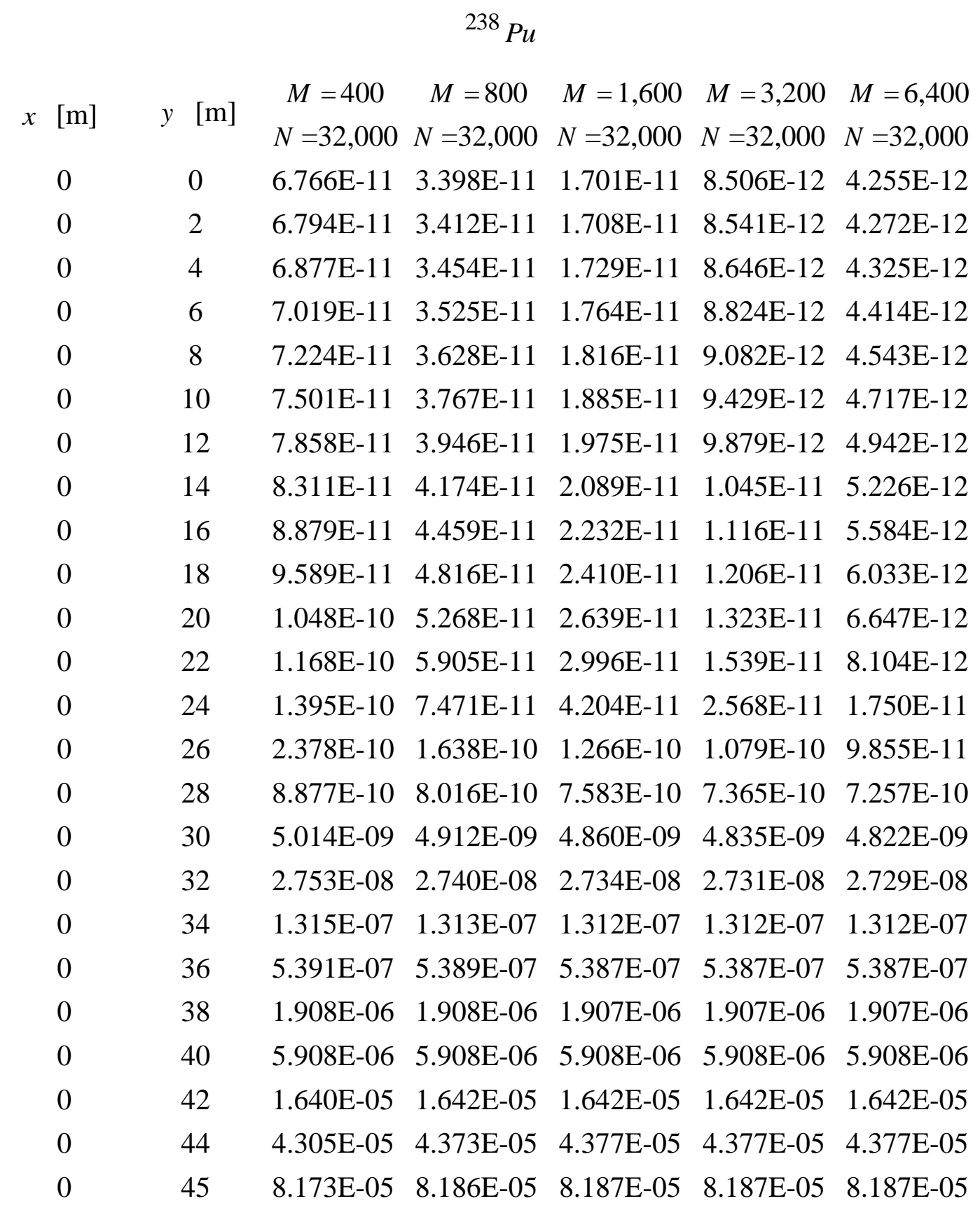


$0 \quad 46 \quad 1.203 \mathrm{E}-04 \quad 1.199 \mathrm{E}-04 \quad 1.198 \mathrm{E}-04 \quad 1.198 \mathrm{E}-04 \quad 1.199 \mathrm{E}-04$

$0 \quad 48 \quad 1.457 \mathrm{E}-04 \quad 1.459 \mathrm{E}-04 \quad 1.459 \mathrm{E}-04 \quad 1.459 \mathrm{E}-04 \quad 1.459 \mathrm{E}-04$

$\begin{array}{lllllll}0 & 50 & 1.522 \mathrm{E}-04 & 1.524 \mathrm{E}-04 & 1.525 \mathrm{E}-04 & 1.525 \mathrm{E}-04 & 1.525 \mathrm{E}-04\end{array}$ 


\begin{tabular}{|c|c|c|c|c|c|c|}
\hline \multirow{2}{*}[\mathrm{m}]{} & \multirow{2}{*}{$\begin{array}{ll}y & {[\mathrm{~m}]}\end{array}$} & $M=6400$ & $M=6400$ & $M=6400$ & $M=6400$ & $M=6400$ \\
\hline & & $N=2,000$ & $N=4,000$ & $N=8,000$ & $N=16,000$ & $N=32,000$ \\
\hline 0 & 0 & $7.852 \mathrm{E}-11$ & $3.538 \mathrm{E}-11$ & $1.718 \mathrm{E}-11$ & 8.527E-12 & $4.255 \mathrm{E}-12$ \\
\hline 0 & 2 & $7.884 \mathrm{E}-11$ & $3.552 \mathrm{E}-11$ & $1.725 \mathrm{E}-11$ & $8.561 \mathrm{E}-12$ & 4.272E-12 \\
\hline 0 & 4 & $7.981 \mathrm{E}-11$ & $3.596 \mathrm{E}-11$ & $1.746 \mathrm{E}-11$ & 8.666E-12 & $4.325 \mathrm{E}-12$ \\
\hline 0 & 6 & $8.145 \mathrm{E}-11$ & $3.670 \mathrm{E}-11$ & $1.782 \mathrm{E}-11$ & $8.845 \mathrm{E}-12$ & $4.414 \mathrm{E}-12$ \\
\hline 0 & 8 & $8.384 \mathrm{E}-11$ & $3.778 \mathrm{E}-11$ & $1.834 \mathrm{E}-11$ & $9.104 \mathrm{E}-12$ & $4.543 \mathrm{E}-12$ \\
\hline 0 & 10 & 8.704E-11 & $3.922 \mathrm{E}-11$ & $1.905 \mathrm{E}-11$ & $9.452 \mathrm{E}-12$ & 4.717E-12 \\
\hline 0 & 12 & $9.119 \mathrm{E}-11$ & $4.109 \mathrm{E}-11$ & $1.995 \mathrm{E}-11$ & $9.902 \mathrm{E}-12$ & 4.942E-12 \\
\hline 0 & 14 & $9.645 \mathrm{E}-11$ & $4.346 \mathrm{E}-11$ & $2.110 \mathrm{E}-11$ & $1.047 \mathrm{E}-11$ & $5.226 \mathrm{E}-12$ \\
\hline 0 & 16 & $1.030 \mathrm{E}-10$ & $4.643 \mathrm{E}-11$ & $2.255 \mathrm{E}-11$ & $1.119 \mathrm{E}-11$ & $5.584 \mathrm{E}-12$ \\
\hline 0 & 18 & $1.113 \mathrm{E}-10$ & $5.014 \mathrm{E}-11$ & $2.435 \mathrm{E}-11$ & $1.209 \mathrm{E}-11$ & $6.033 \mathrm{E}-12$ \\
\hline 0 & 20 & $1.216 \mathrm{E}-10$ & $5.484 \mathrm{E}-11$ & $2.666 \mathrm{E}-11$ & $1.326 \mathrm{E}-11$ & $6.647 \mathrm{E}-12$ \\
\hline 0 & 22 & $1.354 \mathrm{E}-10$ & $6.145 \mathrm{E}-11$ & $3.026 \mathrm{E}-11$ & $1.542 \mathrm{E}-11$ & 8.104E-12 \\
\hline 0 & 24 & $1.604 \mathrm{E}-10$ & $7.740 \mathrm{E}-11$ & $4.238 \mathrm{E}-11$ & $2.572 \mathrm{E}-11$ & $1.750 \mathrm{E}-11$ \\
\hline 0 & 26 & $2.617 \mathrm{E}-10$ & $1.669 \mathrm{E}-10$ & $1.269 \mathrm{E}-10$ & $1.079 \mathrm{E}-10$ & $9.855 \mathrm{E}-11$ \\
\hline 0 & 28 & $9.154 \mathrm{E}-10$ & $8.052 \mathrm{E}-10$ & 7.587E-10 & 7.366E-10 & 7.257E-10 \\
\hline 0 & 30 & $5.047 \mathrm{E}-09$ & $4.916 \mathrm{E}-09$ & 4.861E-09 & $4.835 \mathrm{E}-09$ & 4.822E-09 \\
\hline 0 & 32 & 2.757E-08 & 2.741E-08 & 2.734E-08 & 2.731E-08 & 2.729E-08 \\
\hline 0 & 34 & $1.315 \mathrm{E}-07$ & $1.313 \mathrm{E}-07$ & $1.312 \mathrm{E}-07$ & $1.312 \mathrm{E}-07$ & $1.312 \mathrm{E}-07$ \\
\hline 0 & 36 & 5.391E-07 & 5.389E-07 & 5.387E-07 & 5.387E-07 & 5.387E-07 \\
\hline 0 & 38 & $1.908 \mathrm{E}-06$ & $1.908 \mathrm{E}-06$ & 1.907E-06 & 1.907E-06 & $1.907 \mathrm{E}-06$ \\
\hline 0 & 40 & 5.909E-06 & $5.908 \mathrm{E}-06$ & 5.908E-06 & 5.908E-06 & $5.908 \mathrm{E}-06$ \\
\hline 0 & 42 & $1.642 \mathrm{E}-05$ & $1.642 \mathrm{E}-05$ & $1.642 \mathrm{E}-05$ & $1.642 \mathrm{E}-05$ & $1.642 \mathrm{E}-05$ \\
\hline 0 & 44 & $4.378 \mathrm{E}-05$ & 4.377E-05 & 4.377E-05 & 4.377E-05 & 4.377E-05 \\
\hline 0 & 45 & 8.187E-05 & 8.187E-05 & 8.187E-05 & 8.187E-05 & 8.187E-05 \\
\hline 0 & 46 & $1.198 \mathrm{E}-04$ & 1.198E-04 & 1.198E-04 & 1.198E-04 & 1.199E-04 \\
\hline 0 & 48 & $1.459 \mathrm{E}-04$ & 1.459E-04 & $1.459 \mathrm{E}-04$ & $1.459 \mathrm{E}-04$ & $1.459 \mathrm{E}-04$ \\
\hline 0 & 50 & $1.525 \mathrm{E}-04$ & $1.525 \mathrm{E}-04$ & $1.525 \mathrm{E}-04$ & $1.525 \mathrm{E}-04$ & $1.525 \mathrm{E}-04$ \\
\hline
\end{tabular}


${ }^{238} \mathrm{Pu}$

\begin{tabular}{|c|c|c|c|c|c|c|c|}
\hline [m] & $y \quad[\mathrm{~m}]$ & $\begin{array}{c}M=400 \\
N=2,000\end{array}$ & $\begin{array}{c}M=800 \\
N=4,000\end{array}$ & $\begin{aligned} M & =1,600 \\
N & =8,000\end{aligned}$ & $\begin{array}{l}M=3,200 \\
N=16,000\end{array}$ & $\begin{array}{l}M=6,400 \\
N=32,000\end{array}$ & $\begin{array}{c}M= \\
12,800 \\
N=64,000\end{array}$ \\
\hline 0 & 0 & $1.083 \mathrm{E}-09$ & $2.720 \mathrm{E}-10$ & $6.807 \mathrm{E}-11$ & $1.702 \mathrm{E}-11$ & $4.255 \mathrm{E}-12$ & $1.064 \mathrm{E}-12$ \\
\hline 0 & 2 & $.088 \mathrm{E}-09$ & $2.731 \mathrm{E}-10$ & $6.835 \mathrm{E}-11$ & $1.709 \mathrm{E}-11$ & 4.272E-12 & $1.068 \mathrm{E}-12$ \\
\hline 0 & 4 & 1.101E-09 & $2.765 \mathrm{E}-10$ & $6.918 \mathrm{E}-11$ & $1.730 \mathrm{E}-11$ & $4.325 \mathrm{E}-12$ & $1.081 \mathrm{E}-12$ \\
\hline 0 & 6 & $124 \mathrm{E}-09$ & $2.822 \mathrm{E}-10$ & $7.061 \mathrm{E}-11$ & $1.766 \mathrm{E}-11$ & $4.414 \mathrm{E}-12$ & $1.103 \mathrm{E}-12$ \\
\hline 0 & 8 & $157 \mathrm{E}-09$ & $2.904 \mathrm{E}-10$ & $7.268 \mathrm{E}-11$ & $1.817 \mathrm{E}-11$ & $4.543 \mathrm{E}-12$ & $1.136 \mathrm{E}-12$ \\
\hline 0 & 10 & 201E-09 & $3.015 \mathrm{E}-10$ & E-11 & -11 & -12 & $E-12$ \\
\hline 0 & 12 & $1.258 \mathrm{E}-09$ & $3.159 \mathrm{E}-10$ & $7.905 \mathrm{E}-11$ & $1.977 \mathrm{E}-11$ & 4.942E-12 & $1.235 \mathrm{E}-12$ \\
\hline 0 & 14 & $1.330 \mathrm{E}-09$ & $3.341 \mathrm{E}-10$ & 8.361E-11 & $2.091 \mathrm{E}-11$ & $5.226 \mathrm{E}-12$ & $1.307 \mathrm{E}-12$ \\
\hline 0 & 16 & $.421 \mathrm{E}-09$ & $3.569 \mathrm{E}-10$ & $8.932 \mathrm{E}-11$ & $2.233 \mathrm{E}-11$ & E-12 & $1.396 \mathrm{E}-12$ \\
\hline 0 & 18 & 535E-09 & $3.855 \mathrm{E}-10$ & $9.646 \mathrm{E}-11$ & $2.412 \mathrm{E}-11$ & $6.033 \mathrm{E}-12$ & $1.511 \mathrm{E}-12$ \\
\hline 0 & 20 & $.677 \mathrm{E}-09$ & $4.212 \mathrm{E}-10$ & $1.055 \mathrm{E}-10$ & $2.641 \mathrm{E}-11$ & $6.647 \mathrm{E}-12$ & $1.706 \mathrm{E}-12$ \\
\hline 0 & 22 & $1.857 \mathrm{E}-09$ & $4.670 \mathrm{E}-10$ & $1.175 \mathrm{E}-10$ & $2.998 \mathrm{E}-11$ & 8.104E-12 & $2.634 \mathrm{E}-12$ \\
\hline 0 & 24 & 2.094E-09 & $5.328 \mathrm{E}-10$ & $1.403 \mathrm{E}-10$ & $4.207 \mathrm{E}-11$ & $1.750 \mathrm{E}-11$ & $1.136 \mathrm{E}-11$ \\
\hline 0 & 26 & $2.468 \mathrm{E}-09$ & $6.867 \mathrm{E}-10$ & $2.387 \mathrm{E}-10$ & $1.266 \mathrm{E}-10$ & $9.855 \mathrm{E}-11$ & $9.154 \mathrm{E}-11$ \\
\hline 0 & 28 & $3.482 \mathrm{E}-09$ & $1.410 \mathrm{E}-09$ & $8.887 \mathrm{E}-10$ & $7.583 \mathrm{E}-10$ & E-10 & $7.175 \mathrm{E}-10$ \\
\hline 0 & 30 & 8.100E-09 & $5.635 \mathrm{E}-09$ & $5.016 \mathrm{E}-09$ & 4.860E-09 & 4.822E-09 & 4.812E-09 \\
\hline 0 & 32 & $3.130 \mathrm{E}-08$ & $2.829 \mathrm{E}-08$ & $2.753 \mathrm{E}-08$ & $2.734 \mathrm{E}-08$ & $2.729 \mathrm{E}-08$ & $2.728 \mathrm{E}-08$ \\
\hline 0 & 34 & $1.363 \mathrm{E}-07$ & $1.324 \mathrm{E}-07$ & $1.315 \mathrm{E}-07$ & $1.312 \mathrm{E}-07$ & $1.312 \mathrm{E}-07$ & $1.312 \mathrm{E}-07$ \\
\hline 0 & 36 & $5.454 \mathrm{E}-07$ & $5.403 \mathrm{E}-07$ & 5.391E-07 & $5.387 \mathrm{E}-07$ & $5.387 \mathrm{E}-07$ & $5.386 \mathrm{E}-07$ \\
\hline 0 & 38 & $1.917 \mathrm{E}-06$ & $1.910 \mathrm{E}-06$ & $1.908 \mathrm{E}-06$ & $1.907 \mathrm{E}-06$ & $1.907 \mathrm{E}-06$ & $1.907 \mathrm{E}-06$ \\
\hline 0 & 40 & 5.922E-06 & 5.911E-06 & 5.909E-06 & $5.908 \mathrm{E}-06$ & $5.908 \mathrm{E}-06$ & $5.908 \mathrm{E}-06$ \\
\hline 0 & 42 & $1.643 \mathrm{E}-05$ & $1.642 \mathrm{E}-05$ & $1.642 \mathrm{E}-05$ & $1.642 \mathrm{E}-05$ & $1.642 \mathrm{E}-05$ & $1.642 \mathrm{E}-05$ \\
\hline 0 & 44 & $4.315 \mathrm{E}-05$ & 4.375E-05 & 4.378E-05 & 4.377E-05 & 4.377E-05 & 4.377E-05 \\
\hline 0 & 45 & $8.172 \mathrm{E}-05$ & $8.185 \mathrm{E}-05$ & 8.187E-05 & 8.187E-05 & 8.187E-05 & $8.187 \mathrm{E}-05$ \\
\hline 0 & 46 & $1.202 \mathrm{E}-04$ & $1.198 \mathrm{E}-04$ & $1.198 \mathrm{E}-04$ & 1.198E-04 & 1.199E-04 & $1.199 \mathrm{E}-04$ \\
\hline 0 & 48 & $1.456 \mathrm{E}-04$ & $1.459 \mathrm{E}-04$ & $1.459 \mathrm{E}-04$ & $1.459 \mathrm{E}-04$ & $1.459 \mathrm{E}-04$ & $1.459 \mathrm{E}-04$ \\
\hline$\Omega$ & 50 & $1.521 \mathrm{E}-04$ & $1.524 \mathrm{E}-04$ & $1.525 \mathrm{E}-04$ & $1.525 \mathrm{E}-04$ & $1.525 \mathrm{E}-04$ & $1.525 \mathrm{E}-\mathrm{C}$ \\
\hline
\end{tabular}




$$
{ }^{234} U
$$

\begin{tabular}{|c|c|c|c|c|c|c|}
\hline$x \quad[\mathrm{~m}]$ & $\begin{array}{ll}y & {[\mathrm{~m}]}\end{array}$ & $\begin{array}{c}M=800 \\
N=64,000\end{array}$ & $\begin{array}{l}M=1,600 \\
N=64,000\end{array}$ & $\begin{array}{l}M=3,200 \\
N=64,000\end{array}$ & $\begin{array}{l}M=6,400 \\
N=64,000\end{array}$ & $\begin{array}{c}M= \\
12,800 \\
N=64,000\end{array}$ \\
\hline 0 & 0 & $4.582 \mathrm{E}-08$ & $2.293 \mathrm{E}-08$ & $1.147 \mathrm{E}-08$ & $5.734 \mathrm{E}-09$ & $2.868 \mathrm{E}-09$ \\
\hline 0 & 2 & $4.600 \mathrm{E}-08$ & 2.302E-08 & $1.151 \mathrm{E}-08$ & $5.758 \mathrm{E}-09$ & $2.880 \mathrm{E}-09$ \\
\hline 0 & 4 & $4.657 \mathrm{E}-08$ & $2.331 \mathrm{E}-08$ & $1.165 \mathrm{E}-08$ & $5.828 \mathrm{E}-09$ & $2.915 \mathrm{E}-09$ \\
\hline 0 & 6 & 4.753E-08 & $2.379 \mathrm{E}-08$ & $1.190 \mathrm{E}-08$ & $5.948 \mathrm{E}-09$ & $2.976 \mathrm{E}-09$ \\
\hline 0 & 8 & $4.892 \mathrm{E}-08$ & $2.448 \mathrm{E}-08$ & $1.224 \mathrm{E}-08$ & $6.122 \mathrm{E}-09$ & $3.063 \mathrm{E}-09$ \\
\hline 0 & 10 & $5.079 \mathrm{E}-08$ & $2.542 \mathrm{E}-08$ & $1.271 \mathrm{E}-08$ & 6.357E-09 & 3.180E-09 \\
\hline 0 & 12 & $5.321 \mathrm{E}-08$ & $2.663 \mathrm{E}-08$ & $1.332 \mathrm{E}-08$ & $6.659 \mathrm{E}-09$ & $3.331 \mathrm{E}-09$ \\
\hline 0 & 14 & $5.627 \mathrm{E}-08$ & $2.816 \mathrm{E}-08$ & $1.408 \mathrm{E}-08$ & 7.043E-09 & $3.523 \mathrm{E}-09$ \\
\hline 0 & 16 & $6.012 \mathrm{E}-08$ & $3.009 \mathrm{E}-08$ & $1.505 \mathrm{E}-08$ & 7.524E-09 & 3.764E-09 \\
\hline 0 & 18 & $6.492 \mathrm{E}-08$ & $3.249 \mathrm{E}-08$ & $1.625 \mathrm{E}-08$ & $8.126 \mathrm{E}-09$ & $4.065 \mathrm{E}-09$ \\
\hline 0 & 20 & 7.094E-08 & $3.551 \mathrm{E}-08$ & $1.776 \mathrm{E}-08$ & 8.880E-09 & 4.442E-09 \\
\hline 0 & 22 & $7.854 \mathrm{E}-08$ & $3.932 \mathrm{E}-08$ & $1.968 \mathrm{E}-08$ & $9.850 \mathrm{E}-09$ & 4.939E-09 \\
\hline 0 & 24 & 8.863E-08 & 4.459E-08 & $2.253 \mathrm{E}-08$ & $1.150 \mathrm{E}-08$ & $5.984 \mathrm{E}-09$ \\
\hline 0 & 26 & $1.084 \mathrm{E}-07$ & $5.817 \mathrm{E}-08$ & $3.299 \mathrm{E}-08$ & $2.040 \mathrm{E}-08$ & $1.410 \mathrm{E}-08$ \\
\hline 0 & 28 & $2.247 \mathrm{E}-07$ & $1.662 \mathrm{E}-07$ & $1.370 \mathrm{E}-07$ & $1.223 \mathrm{E}-07$ & $1.150 \mathrm{E}-07$ \\
\hline 0 & 30 & $1.347 \mathrm{E}-06$ & $1.278 \mathrm{E}-06$ & $1.243 \mathrm{E}-06$ & $1.225 \mathrm{E}-06$ & $1.217 \mathrm{E}-06$ \\
\hline 0 & 32 & $1.114 \mathrm{E}-05$ & $1.106 \mathrm{E}-05$ & $1.102 \mathrm{E}-05$ & $1.100 \mathrm{E}-05$ & $1.098 \mathrm{E}-05$ \\
\hline 0 & 34 & 8.089E-05 & $8.078 \mathrm{E}-05$ & $8.073 \mathrm{E}-05$ & $8.070 \mathrm{E}-05$ & $8.069 \mathrm{E}-05$ \\
\hline 0 & 36 & 4.817E-04 & $4.815 \mathrm{E}-04$ & $4.815 \mathrm{E}-04$ & $4.814 \mathrm{E}-04$ & 4.814E-04 \\
\hline 0 & 38 & $2.348 \mathrm{E}-03$ & $2.348 \mathrm{E}-03$ & $2.348 \mathrm{E}-03$ & $2.348 \mathrm{E}-03$ & $2.348 \mathrm{E}-03$ \\
\hline 0 & 40 & $9.480 \mathrm{E}-03$ & $9.480 \mathrm{E}-03$ & $9.479 \mathrm{E}-03$ & $9.479 \mathrm{E}-03$ & $9.479 \mathrm{E}-03$ \\
\hline 0 & 42 & $3.248 \mathrm{E}-02$ & $3.248 \mathrm{E}-02$ & $3.248 \mathrm{E}-02$ & $3.248 \mathrm{E}-02$ & $3.248 \mathrm{E}-02$ \\
\hline 0 & 44 & $1.011 \mathrm{E}-01$ & $1.012 \mathrm{E}-01$ & $1.012 \mathrm{E}-01$ & $1.012 \mathrm{E}-01$ & $1.012 \mathrm{E}-01$ \\
\hline 0 & 45 & $2.024 \mathrm{E}-01$ & $2.025 \mathrm{E}-01$ & $2.025 \mathrm{E}-01$ & $2.025 \mathrm{E}-01$ & $2.025 \mathrm{E}-01$ \\
\hline 0 & 46 & $3.035 \mathrm{E}-01$ & $3.035 \mathrm{E}-01$ & $3.035 \mathrm{E}-01$ & $3.035 \mathrm{E}-01$ & $3.035 \mathrm{E}-01$ \\
\hline 0 & 48 & $3.704 \mathrm{E}-01$ & $3.705 \mathrm{E}-01$ & $3.705 \mathrm{E}-01$ & $3.705 \mathrm{E}-01$ & $3.705 \mathrm{E}-01$ \\
\hline 0 & 50 & $3.862 \mathrm{E}-01$ & $3.864 \mathrm{E}-01$ & $3.864 \mathrm{E}-01$ & $3.864 \mathrm{E}-01$ & $3.864 \mathrm{E}-01$ \\
\hline
\end{tabular}




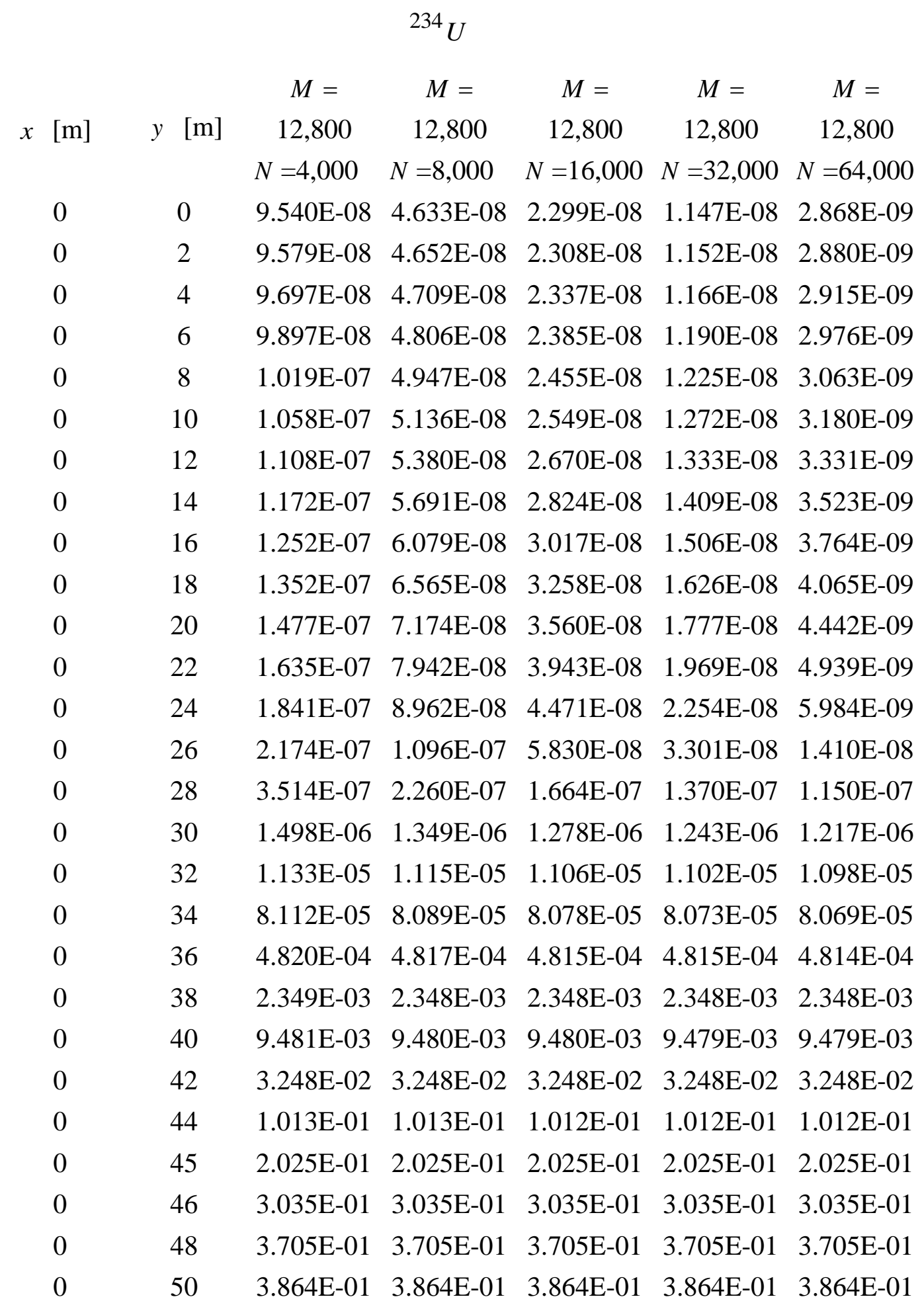




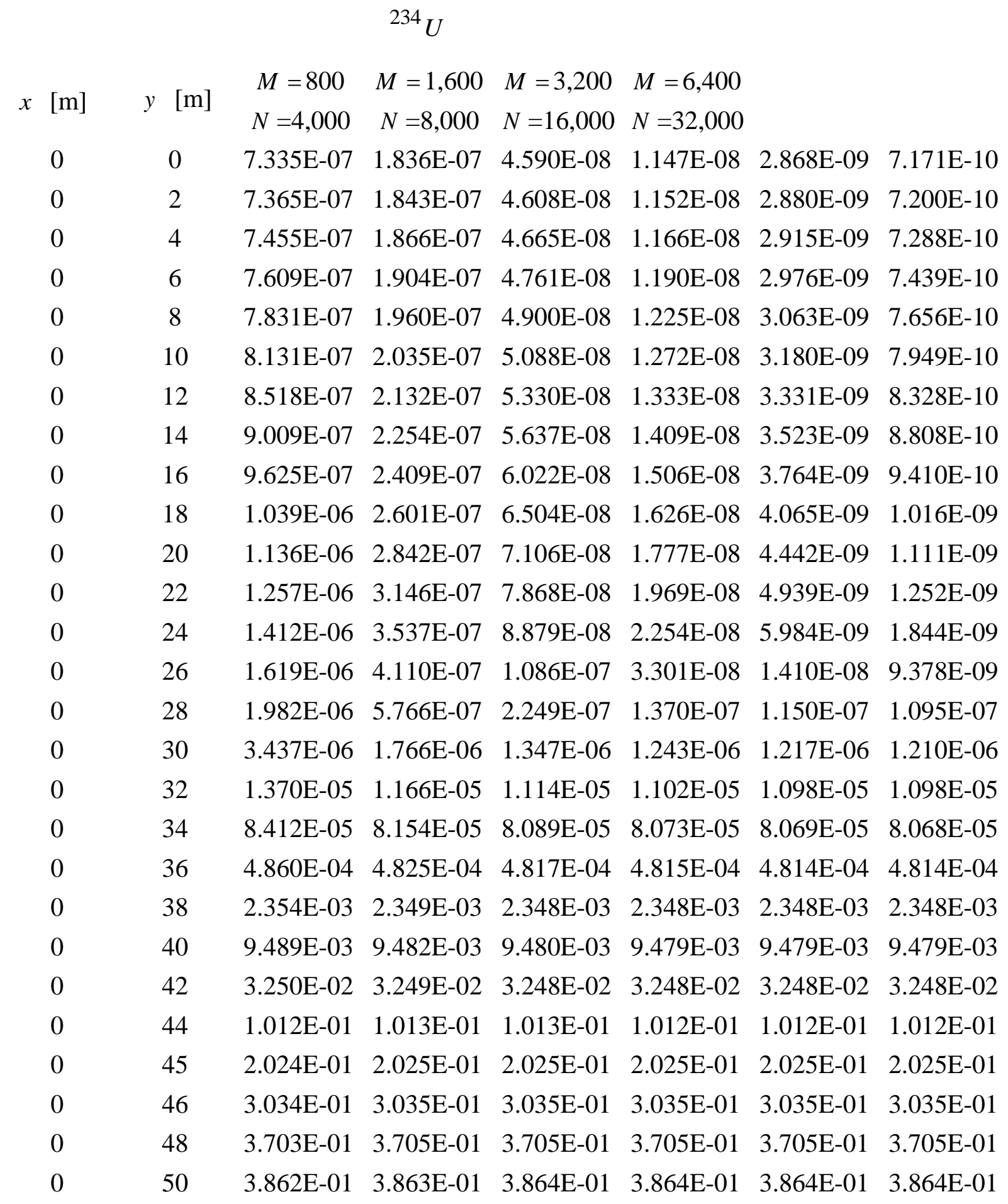




$\begin{array}{lccccccc}x[\mathrm{~m}] & y & {[\mathrm{~m}]} & M=800 & & & & \\ 0 & 0 & 6.622 \mathrm{E}-10 & 3.326 \mathrm{E}-10 & 1.664 \mathrm{E}-10 & 8.325 \mathrm{E}-11 & 4.164 \mathrm{E}-11 \\ 0 & 2 & 6.649 \mathrm{E}-10 & 3.339 \mathrm{E}-10 & 1.671 \mathrm{E}-10 & 8.358 \mathrm{E}-11 & 4.181 \mathrm{E}-11 \\ 0 & 4 & 6.730 \mathrm{E}-10 & 3.380 \mathrm{E}-10 & 1.692 \mathrm{E}-10 & 8.461 \mathrm{E}-11 & 4.232 \mathrm{E}-11 \\ 0 & 6 & 6.869 \mathrm{E}-10 & 3.450 \mathrm{E}-10 & 1.727 \mathrm{E}-10 & 8.635 \mathrm{E}-11 & 4.320 \mathrm{E}-11 \\ 0 & 8 & 7.070 \mathrm{E}-10 & 3.551 \mathrm{E}-10 & 1.777 \mathrm{E}-10 & 8.888 \mathrm{E}-11 & 4.446 \mathrm{E}-11 \\ 0 & 10 & 7.341 \mathrm{E}-10 & 3.687 \mathrm{E}-10 & 1.845 \mathrm{E}-10 & 9.228 \mathrm{E}-11 & 4.616 \mathrm{E}-11 \\ 0 & 12 & 7.690 \mathrm{E}-10 & 3.862 \mathrm{E}-10 & 1.933 \mathrm{E}-10 & 9.668 \mathrm{E}-11 & 4.836 \mathrm{E}-11 \\ 0 & 14 & 8.133 \mathrm{E}-10 & 4.085 \mathrm{E}-10 & 2.044 \mathrm{E}-10 & 1.022 \mathrm{E}-10 & 5.115 \mathrm{E}-11 \\ 0 & 16 & 8.689 \mathrm{E}-10 & 4.364 \mathrm{E}-10 & 2.184 \mathrm{E}-10 & 1.092 \mathrm{E}-10 & 5.464 \mathrm{E}-11 \\ 0 & 18 & 9.384 \mathrm{E}-10 & 4.713 \mathrm{E}-10 & 2.359 \mathrm{E}-10 & 1.180 \mathrm{E}-10 & 5.901 \mathrm{E}-11 \\ 0 & 20 & 1.025 \mathrm{E}-09 & 5.149 \mathrm{E}-10 & 2.577 \mathrm{E}-10 & 1.289 \mathrm{E}-10 & 6.448 \mathrm{E}-11 \\ 0 & 22 & 1.135 \mathrm{E}-09 & 5.700 \mathrm{E}-10 & 2.853 \mathrm{E}-10 & 1.427 \mathrm{E}-10 & 7.137 \mathrm{E}-11 \\ 0 & 24 & 1.274 \mathrm{E}-09 & 6.400 \mathrm{E}-10 & 3.203 \mathrm{E}-10 & 1.602 \mathrm{E}-10 & 8.017 \mathrm{E}-11 \\ 0 & 26 & 1.455 \mathrm{E}-09 & 7.311 \mathrm{E}-10 & 3.662 \mathrm{E}-10 & 1.835 \mathrm{E}-10 & 9.209 \mathrm{E}-11 \\ 0 & 28 & 1.702 \mathrm{E}-09 & 8.597 \mathrm{E}-10 & 4.353 \mathrm{E}-10 & 2.227 \mathrm{E}-10 & 1.164 \mathrm{E}-10 \\ 0 & 30 & 2.146 \mathrm{E}-09 & 1.144 \mathrm{E}-09 & 6.395 \mathrm{E}-10 & 3.866 \mathrm{E}-10 & 2.602 \mathrm{E}-10 \\ 0 & 32 & 3.929 \mathrm{E}-09 & 2.704 \mathrm{E}-09 & 2.087 \mathrm{E}-09 & 1.778 \mathrm{E}-09 & 1.624 \mathrm{E}-09 \\ 0 & 34 & 1.644 \mathrm{E}-08 & 1.489 \mathrm{E}-08 & 1.411 \mathrm{E}-08 & 1.372 \mathrm{E}-08 & 1.352 \mathrm{E}-08 \\ 0 & 36 & 1.043 \mathrm{E}-07 & 1.023 \mathrm{E}-07 & 1.013 \mathrm{E}-07 & 1.007 \mathrm{E}-07 & 1.005 \mathrm{E}-07 \\ 0 & 38 & 6.366 \mathrm{E}-07 & 6.337 \mathrm{E}-07 & 6.323 \mathrm{E}-07 & 6.316 \mathrm{E}-07 & 6.312 \mathrm{E}-07 \\ 0 & 40 & 3.429 \mathrm{E}-06 & 3.427 \mathrm{E}-06 & 3.424 \mathrm{E}-06 & 3.423 \mathrm{E}-06 & 3.423 \mathrm{E}-06 \\ 0 & 42 & 1.773 \mathrm{E}-05 & 1.783 \mathrm{E}-05 & 1.782 \mathrm{E}-05 & 1.782 \mathrm{E}-05 & 1.782 \mathrm{E}-05 \\ 0 & 44 & 1.028 \mathrm{E}-04 & 1.089 \mathrm{E}-04 & 1.093 \mathrm{E}-04 & 1.093 \mathrm{E}-04 & 1.093 \mathrm{E}-04 \\ 0 & 45 & 3.639 \mathrm{E}-04 & 3.636 \mathrm{E}-04 & 3.637 \mathrm{E}-04 & 3.637 \mathrm{E}-04 & 3.637 \mathrm{E}-04 \\ 0 & 46 & 6.249 \mathrm{E}-04 & 6.183 \mathrm{E}-04 & 6.180 \mathrm{E}-04 & 6.181 \mathrm{E}-04 & 6.181 \mathrm{E}-04 \\ 0 & 48 & 7.095 \mathrm{E}-04 & 7.089 \mathrm{E}-04 & 7.090 \mathrm{E}-04 & 7.090 \mathrm{E}-04 & 7.090 \mathrm{E}-04 \\ 0 & 50 & 7.210 \mathrm{E}-04 & 7.205 \mathrm{E}-04 & 7.206 \mathrm{E}-04 & 7.206 \mathrm{E}-04 & 7.206 \mathrm{E}-04\end{array}$


